\title{
Synergistic Effects of Transplanted Adult Neural Stem/Progenitor Cells, Chondroitinase, and Growth Factors Promote Functional Repair and Plasticity of the Chronically Injured Spinal Cord
}

\author{
Soheila Karimi-Abdolrezaee, ${ }^{1,2,3,4,6}$ Eftekhar Eftekharpour, ${ }^{1}$ Jian Wang, ${ }^{1}$ Desiree Schut, ${ }^{1}$ and Michael G. Fehlings ${ }^{1,2,3,5,6}$ \\ ${ }^{1}$ Division of Genetics and Development, Toronto Western Research Institute and Krembil Neuroscience Center, University Health Network, Toronto, \\ Ontario M5T 2S8, Canada, and ${ }^{2}$ Department of Surgery, Divisions of ${ }^{3}$ Neurosurgery and ${ }^{4}$ Anatomy, ${ }^{5}$ Institute of Medical Science, and ${ }^{6}$ Neuroscience \\ Program, University of Toronto, Toronto, Ontario M5S 1A8, Canada
}

The transplantation of neural stem/progenitor cells (NPCs) is a promising therapeutic strategy for spinal cord injury (SCI). However, to date NPC transplantation has exhibited only limited success in the treatment of chronic SCI. Here, we show that chondroitin sulfate proteoglycans (CSPGs) in the glial scar around the site of chronic SCI negatively influence the long-term survival and integration of transplanted NPCs and their therapeutic potential for promoting functional repair and plasticity. We targeted CSPGs in the chronically injured spinal cord by sustained infusion of chondroitinase $\mathrm{ABC}(\mathrm{ChABC})$. One week later, the same rats were treated with transplants of NPCs and transient infusion of growth factors, EGF, bFGF, and PDGF-AA. We demonstrate that perturbing CSPGs dramatically optimizes NPC transplantation in chronic SCI. Engrafted NPCs successfully integrate and extensively migrate within the host spinal cord and principally differentiate into oligodendrocytes. Furthermore, this combined strategy promoted the axonal integrity and plasticity of the corticospinal tract and enhanced the plasticity of descending serotonergic pathways. These neuroanatomical changes were also associated with significantly improved neurobehavioral recovery after chronic SCI. Importantly, this strategy did not enhance the aberrant synaptic connectivity of pain afferents, nor did it exacerbate posttraumatic neuropathic pain. For the first time, we demonstrate key biological and functional benefits for the combined use of ChABC, growth factors, and NPCs to repair the chronically injured spinal cord. These findings could potentially bring us closer to the application of NPCs for patients suffering from chronic SCI or other conditions characterized by the formation of a glial scar.

\section{Introduction}

Repair of the chronically injured spinal cord is inherently challenging due to multiple factors, including cellular loss, a cystic cavity that disrupts axonal pathways, and the inhibitory influence of the glial scar (Silver and Miller, 2004; Thuret et al., 2006). Therefore, treatment of chronic spinal cord injury (SCI) will require a multifaceted strategy. To date, no successful functional treatment for contusive chronic SCI has been achieved.

\footnotetext{
Received June 26, 2009; revised Dec. 7, 2009; accepted Dec. 12, 2009.

This work was supported by operating grants from the Christopher and Dana Reeve Foundation and the Sam Schmidt Paralysis Foundation to S.K.-A. and M.G.F., AOSpine North America to S.K.-A. and M.G.F., and the Canadian Institutes of Health Research and Krembil Foundation to M.G.F. We thank Behzad Azad for his contribution to anima care and neurobehavioral assessments, as well as Sarah Chan, Mehdi Afshar, and Glen Viola for their assistance in the histological and neurobehavioral assessments.

Correspondence should be addressed to either of the following: Dr. Soheila Karimi-Abdolrezaee, Department of Physiology and the Regenerative Medicine Program, Faculty of Medicine, University of Manitoba, 745 Bannatyne Avenue, Room 435 BMSB, Winnipeg, MB R3E 0J9, Canada, E-mail: karimis@cc.umanitoba.ca; or Dr. Michael G. Fehlings, Krembil Chair in Neural Repair and Regeneration, Department of Surgery, Division of Neurosurgery, University of Toronto, and Toronto Western Hospital, University Health Network, Room 4W-449, 399 Bathurst Street, Toronto, 0N M5T 2S8, Canada, E-mail: michael.fehlings@uhn.on.ca.

DOI:10.1523/JNEUROSCI.3111-09.2010

Copyright $\odot 2010$ the authors $\quad 0270-6474 / 10 / 301657-20 \$ 15.00 / 0$
}

Transplantation of neural precursor cells (NPCs) holds great promise for SCI repair and regeneration (Cummings et al., 2005; Goldman, 2005; Hofstetter et al., 2005; Karimi-Abdolrezaee et al., 2006; Ziv et al., 2006; Parr et al., 2008). NPCs are the somatic stem cells of the CNS, which are characterized by the capacity for selfrenewal and multilineage potential (Reynolds and Weiss, 1992; Morshead et al., 1994; Alvarez-Buylla and Temple, 1998; Horner et al., 2000). Additionally, NPCs may exert tropic or trophic effects that could enhance axonal sparing, plasticity, and possibly regeneration (Teng et al., 2002; Lu et al., 2003; Teng et al., 2006). Recently, we developed a NPC-cell-based strategy that combined NPC transplants with in vivo delivery of growth factors (GFs) that markedly increased the long-term survival of NPCs and directed their fate toward an oligodendrocyte lineage, promoted axonal remyelination, and significantly enhanced functional recovery in the subacute phase after SCI (Karimi-Abdolrezaee et al., 2006). However, our findings showed that the time window for the successful application of NPCs after injury was narrow, as the integration of NPCs in the chronically injured spinal cord was very limited (Karimi-Abdolrezaee et al., 2006).

Our data suggested a strong association between the expression of chondroitin sulfate proteoglycans (CSPGs) and the poor 
survival of transplanted NPCs in the chronically injured spinal cord. CSPGs, which are upregulated after injury (Asher et al., 2000; Morgenstern et al., 2002; Jones et al., 2003; Tang et al., 2003), are known to play a key role in hindering regeneration and plasticity after SCI (Bradbury et al., 2002; Silver and Miller, 2004; Barritt et al., 2006; Massey et al., 2006; Cafferty et al., 2007). Moreover, the inhibition of CSPGs promotes neural plasticity and functional recovery after acute or subacute SCI (Bradbury et al., 2002; Fouad et al., 2005; Barritt et al., 2006; Massey et al., 2006, 2008).

Here, in a chronic model of compressive SCI in rodents, we disturbed CSPGs with intrathecal infusion of chondroitinase $A B C$ (ChABC) for $7 \mathrm{~d}$. Then, we transplanted the same chronically injured rats with NPCs and transiently supplemented the spinal cord with the intrathecal infusion of a GF cocktail containing epidermal growth factors (EGF), fibroblast growth factor (bFGF), and plateletderived growth factor (PDGF-AA). We provide strong evidence that this combinatorial approach markedly increases the long-term survival of NPCs and greatly optimizes their migration and integration in the chronically injured spinal cord. Furthermore, we demonstrate multiple mechanisms by which this combinatorial strategy facilitates neuroanatomical plasticity of the chronically injured spinal with improved locomotor recovery and without promoting aberrant plasticity of spinal cord pain pathways or exacerbating posttraumatic neuropathic pain.

\section{Materials and Methods}

All the neurobehavioral and neuroanatomical protocols in this study were performed using appropriate randomization and blinding. Unbiased stereological approaches were used to undertake the neuroanatomical assessments.

\section{Animal care}

A total of 165 adult female Wistar rats (250 g; Charles River Laboratories) were used for different aspects of this study. All experimental protocols were approved by the animal care committee of the University Health Network in accordance with the policies established in the guide to the care and use of experimental animals prepared by the Canadian Council of Animal Care.

\section{Isolation and culturing of adult neural precursor cells}

Adult NPCs were isolated from YFP-expressing transgenic mice [strain name: $129-\mathrm{Tg}$ (ACTB-EYFP) 2Nagy/J, The Jackson Laboratory] as previously described (Tropepe et al., 1999; Karimi-Abdolrezaee et al., 2006; Eftekharpour et al., 2007). Briefly, mice were killed by cervical dislocation, and the brains were excised under sterile conditions and transferred to artificial CSF (aCSF) solution containing $2 \mathrm{M} \mathrm{NaCl}, 1 \mathrm{M} \mathrm{KCl}, 1 \mathrm{M}$ $\mathrm{MgCl}_{2}, 155 \mathrm{~mm} \mathrm{NaHCO} 3,108 \mathrm{~mm} \mathrm{CaCl}_{2}, 1 \mathrm{M}$ glucose, and $1 \%$ penicillin/ streptomycin (Pen/Strep) (Sigma). The subventricular zone of forebrain was dissected and transferred to a low-calcium aCSF solution $(10 \mathrm{ml})$ containing $40 \mathrm{mg}$ of trypsin, $20 \mathrm{mg}$ of hyaluronidase, and $4 \mathrm{mg}$ of kynurenic acid for $30 \mathrm{~min}$ at $37^{\circ} \mathrm{C}$. Then, trypsin was inactivated, and the tissue was mechanically dissociated into a cell suspension with a firepolished Pasteur pipette. Cells were plated in uncoated tissue culture flasks in serum-free medium $(200 \mathrm{ml})$ containing $20 \mathrm{ml}$ of DMEM/F12, 4 $\mathrm{ml}$ of $30 \%$ glucose, $3 \mathrm{ml}$ of $7.5 \% \mathrm{NaHCO}_{3}, 1 \mathrm{ml}$ of $1 \mathrm{~m}$ HEPES, $200 \mathrm{mg}$ of transferrin, $50 \mathrm{mg}$ of insulin, $19.25 \mathrm{mg}$ of putrescine, $20 \mu \mathrm{l}$ of selenium, $20 \mu \mathrm{l}$ of progesterone, $1 \mu \mathrm{g}$ of FGF2, $2 \mu \mathrm{g}$ of EGF, and 1\% Pen/Strep for $7 \mathrm{~d}$. The neurospheres generated were passaged weekly by mechanical dissociation in the same medium (as above).

\section{Experimental groups and surgical procedures}

\section{Thoracic spinal cord injury}

The aneurysm clip compression model of SCI used here has been extensively characterized as we have reported previously (Rivlin and Tator, 1978; Fehlings and Tator, 1995; Nashmi and Fehlings, 2001; KarimiAbdolrezaee et al., 2004, 2006). Under halothane anesthesia (1-2\%) and a 1:1 mixture of $\mathrm{O}_{2} / \mathrm{N}_{2} \mathrm{O}$, the surgical area was shaved and disinfected with $70 \%$ ethanol and betadine. A midline incision was made at the thoracic area (T4-T9), and skin and superficial muscles were retracted. The rats underwent a T6-T8 laminectomy and then received a 23 g clip (Walsh) compression injury for $1 \mathrm{~min}$ at the level of T7 of the spinal cord. The surgical wounds were sutured, and the animals were given postoperative analgesia and saline $(0.9 \% ; 5 \mathrm{ml})$ to prevent dehydration and received Clavamox (amoxicillin plus clavulanic acid) for $7 \mathrm{~d}$. Animals were allowed to recover and housed in standard rat cages with absorbent bedding at a temperature of $26^{\circ} \mathrm{C}$. Their bladders were manually expressed three times daily until return of reflexive bladder control.

\section{Experimental groups and treatments}

At 6 weeks after injury, all injured rats were block randomized into five experimental groups based on their Basso, Beattie, and Bresnahan (BBB) open field locomotor score (see below, neurobehavioral assessments) to ensure equivalent deficits across the experimental groups before starting the treatments. A summary of the experimental protocol is depicted in Table 1.

ChABC treatment. At 6 weeks after injury, the rats were anesthetized using halothane inhalation $(1-2 \%)$ and a $1: 1$ mixture of $\mathrm{O}_{2} / \mathrm{N}_{2} \mathrm{O}$, and then the injured spinal cord was carefully reexposed using microsurgical techniques. ChABC (Seikagaku, $5 \mathrm{U} / \mathrm{ml}$ in saline plus $0.1 \%$ rat serum albumin) was administered intrathecally using a catheter [Alzet, Rat IT, 0007741, $0.36 \mathrm{~mm}$ outer diameter (OD); $0.18 \mathrm{~mm}$ inner diameter (ID)] connected to an osmotic mini-pump (Alzet pump model No. 1007D, 0.5 $\mu \mathrm{l} / \mathrm{h}$ ) for $7 \mathrm{~d}$. The catheter was inserted in the subarachnoid space around the injured area. Rats in other experimental groups except the plain injured underwent identical surgical procedures but received vehicle (penicillinase in saline plus $0.1 \%$ rat serum albumin).

Transplantation of adult NPCs and administration of growth factor treatment. One week after ChABC or vehicle treatment ( 7 weeks after SCI), the animals underwent intraspinal injections of NPCs or vehicle using well established methods developed by our group (KarimiAbdolrezaee et al., 2006; Eftekharpour et al., 2007). Briefly, the injured rats were anesthetized using halothane inhalation (1-2\%) and a 1:1 mixture of $\mathrm{O}_{2} / \mathrm{N}_{2} \mathrm{O}$, and then the injured spinal cord was carefully reexposed using microsurgical techniques. For NPC transplantation, the rats were stereotactically injected with a cell suspension of adult NPCs. Assessment of cell viability by Trypan Blue staining indicated the presence of $\sim 90 \%$ live cells in the cell suspension. The cells were diluted in the growth medium $\left(50 \times 10^{3}\right.$ live cells $\left./ \mu \mathrm{l}\right)$ and then used for cell transplantation. Using a Hamilton syringe connected to a microglass pipette $(100 \mu \mathrm{m}$ OD), a total volume of $8 \mu \mathrm{l}$ of cell suspension, containing $4 \times 10^{5}$ live cells, was intraspinally injected into the dorsolateral spinal cord, next to the midline. Four intraspinal injections were bilaterally made at a point 2 $\mathrm{mm}$ rostral and $2 \mathrm{~mm}$ caudal to the injury site. To enhance the survival of the transplanted cells and to direct the fate of NPCs along an oligodendroglial lineage, a cocktail of GFs including PDGF-AA (Sigma, $1 \mu \mathrm{g} / 100$ $\mu \mathrm{l}$ ), bFGF (Sigma, $3 \mu \mathrm{g} / 100 \mu \mathrm{l}$ ) and EGF (Sigma, $3 \mu \mathrm{g} / 100 \mu \mathrm{l}$ ) in a solution containing artificial CSF, BSA $(100 \mu \mathrm{g} / \mathrm{ml})$, and gentamicin $(50$ $\mu \mathrm{g} / \mathrm{ml}$ ) was infused for $7 \mathrm{~d}$ using a subarachnoid catheter (Alzet, Rat IT, 0007741, $0.36 \mathrm{~mm}$ OD; $0.18 \mathrm{~mm}$ ID) connected to an osmotic minipump and located near the injury site (Alzet pump model No. 1007D, $0.5 \mu \mathrm{l} / \mathrm{h}$ ), as we have previously reported (Karimi-Abdolrezaee et al., 2006; Eftekharpour et al., 2007). Therefore, GF delivery at the time of NPC transplantation is part of our transplantation protocol. The animals received a daily subcutaneous injection of cyclosporine A (10 mg/kg, Sandimmune, Novartis) starting $3 \mathrm{~d}$ before transplantation and continuing until the end of the experiments. The rats also received a daily injection of the anti-inflammatory drug minocycline ( $50 \mathrm{mg} / \mathrm{kg}$, Sigma) intraperitoneally for $10 \mathrm{~d}$ starting $3 \mathrm{~d}$ before transplantation. Note that animals in all other groups, except the "plain injured" rats (the injury baseline control), also received the same number of injections to the spinal cord using only culture medium, and received cyclosporine $\mathrm{A}$ and minocycline in the same manner as the NPC-transplanted rats.

\section{Biotinylated dextran amine anterograde tracing of the corticospinal tract}

Two weeks before the end of the experiments, rats underwent anterograde tracing of the corticospinal tract (CST) with biotinylated dextran 
Table 1. A summary of experimental timeline and groups

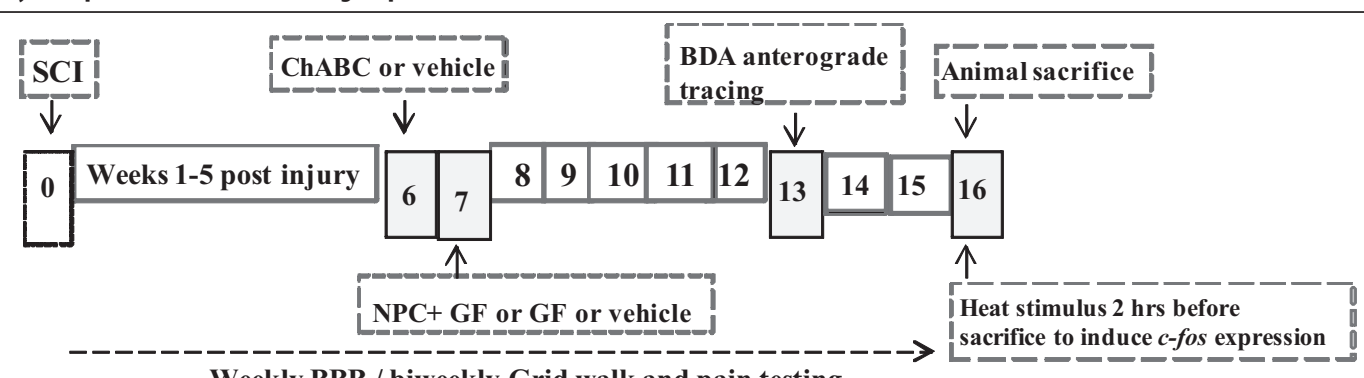

Weekly BBB / biweekly Grid walk and pain testing

SCI: spinal cord injury

ChABC: Chrondroitinase ABC

BDA: Biotinylated Dextran Amine

BBB: Basso, Beattie and Bresnahan Open Field Locomotor Test
NPCs: Neural precursor cells

GFs: Growth factors

Description of the experimental groups and the received treatments

\begin{tabular}{|c|c|c|c|c|c|c|}
\hline Groups & SCI & ChABC & GFs & $\begin{array}{c}\text { NPC } \\
\text { transplant }\end{array}$ & Minocycline & Cyclosporine A \\
\hline $\begin{array}{l}\text { Plain Injured (SCI base- } \\
\text { line }\end{array}$ & + & - & - & - & - & - \\
\hline ChABC & + & + & - & - & + & + \\
\hline Growth Factors (GFs) & + & - & + & - & + & + \\
\hline NPCs + GFs & + & - & + & + & + & + \\
\hline ChABC / NPCs + GFs & + & + & + & + & + & + \\
\hline Un-injured & - & - & - & - & - & - \\
\hline
\end{tabular}

Number of animals that were used for the 16-week period of behavioral assessments:

\begin{tabular}{|c|c|c|c|}
\hline Group & $\begin{array}{l}\text { Group size } \\
\text { at week five } \\
\text { (beginning of the } \\
\text { interventions) }\end{array}$ & $\begin{array}{l}\text { Mortality } \\
\text { (\# of rats) }\end{array}$ & $\begin{array}{l}\text { Number of } \\
\text { animals that } \\
\text { were included in } \\
\text { the assessments }\end{array}$ \\
\hline Plain Injured & 14 & 1 (was euthanized because of a skin lesion) & 13 \\
\hline ChABC & 15 & 2 (died during the fourth surgical procedure for BDA injection) & 13 \\
\hline GFs & 16 & 1 (died during the fourth surgical procedure for BDA injection) & 15 \\
\hline NPCs + GFs & 14 & 0 & 14 \\
\hline ChABC / NPCs + GFs & 15 & 1 (was euthanized because of a skin lesion) & 14 \\
\hline
\end{tabular}

Number of animals that were used in the pilot studies to establish the experimental protocols or other short-term assessments:

\begin{tabular}{|l|c|}
\hline \multicolumn{1}{|c|}{ Group } & Number of rats \\
\hline $\begin{array}{l}\text { Vehicle Injured } \\
\text { (received penicillinase and saline as a } \\
\text { control for ChABC treatment) }\end{array}$ & 14 \\
\hline ChABC & 18 \\
\hline GFs & 12 \\
\hline NPCs + GFs & 16 \\
\hline ChABC / NPCs + GFs & 22 \\
\hline Un-injured & 14 \\
\hline
\end{tabular}

amine (BDA) ( $n=6 /$ group). Under halothane anesthesia (as above), rats were positioned in a stereotaxic frame. BDA (10\%, 10,000 MW; Invitrogen) was injected unilaterally into the left sensorimotor cortex at eight sites $(0.5 \mu \mathrm{l}$ per site) using the following coordinates (in reference to bregma): (1) $1 \mathrm{~mm}$ anterior and $1 \mathrm{~mm}$ lateral; (2) $0.5 \mathrm{~mm}$ anterior and 1 mm lateral; (3) $1 \mathrm{~mm}$ posterior and $1 \mathrm{~mm}$ lateral; (4) $2.5 \mathrm{~mm}$ posterior and $1 \mathrm{~mm}$ lateral; (5) $0.5 \mathrm{~mm}$ posterior and $2 \mathrm{~mm}$ lateral; (6) $1.5 \mathrm{~mm}$ posterior and $2 \mathrm{~mm}$ lateral, (7) $1 \mathrm{~mm}$ anterior and $2.5 \mathrm{~mm}$ lateral, (8) 1.5 $\mathrm{mm}$ anterior and $2.5 \mathrm{~mm}$ lateral. Injections were made $1.2 \mathrm{~mm}$ from the surface of the cortex.

Induction of $c$-fos

On the day of animal perfusion, a subgroup of rats in all experimental groups ( $n=6 /$ group) received a noxious heat stimulus to the der- matomes caudal to the lesion site. This was undertaken to examine the synaptic connectivity of afferents terminating in the superficial layers of dorsal horn by assessing the expression of the immediate early gene $c$-fos. Rats were anesthetized with sodium pentobarbital ( $65 \mathrm{mg} / \mathrm{kg}$, i.p.). Then, each rat received a cutaneous heat stimulus for $60 \mathrm{~s}$ using a hot rock $\left(52^{\circ} \mathrm{C}\right)$. This stimulus was shown to be painful when applied to alert rats during our routine pain test analysis. Two hours after stimulus onset, the rats were perfused as described below.

\section{Tissue processing}

Animal perfusion

Animals were deeply anesthetized with sodium pentobarbital $(80 \mathrm{mg} / \mathrm{kg}$, i.p.) and then perfused transcardially with cold PBS ( $0.1 \mathrm{M})$ followed by 
$4 \%$ paraformaldehyde (PFA) in $0.1 \mathrm{~m}$ PBS, $\mathrm{pH}$ 7.4. A $3 \mathrm{~cm}$ length of the spinal cord centered at the injury center was dissected and processed for different procedures as follows.

Frozen sections. For cryotomy, the spinal cord were postfixed in the perfusing solution plus $10 \%$ sucrose overnight at $4^{\circ} \mathrm{C}$, and then cryoprotected in $20 \%$ sucrose in PBS for $48 \mathrm{~h}$ at $4^{\circ} \mathrm{C}$. Then, the spinal cord centered at the injury site was dissected and embedded in mounting media (HistoPrep, Fisher Scientific) on dry ice. Cryostat sections (30 $\mu \mathrm{m})$ were cut and stored at $-70^{\circ} \mathrm{C}$.

Vibratome sectioning. The spinal cords of the rats that underwent BDA anterograde labeling were postfixed in $4 \%$ PFA for overnight at $4{ }^{\circ} \mathrm{C}$, and then stored in PBS containing $0.1 \%$ sodium azide. The spinal cord were embedded in $5 \%$ agarose (Invitrogen), and $50 \mu \mathrm{m}$ free-floating serial transverse sections were cut on a vibratome and collected in 24-well plates containing PBS plus $0.1 \%$ sodium azide.

\section{Immunohistochemical procedures and image analysis}

For all immunohistochemical staining, the blocking solution contained $5 \%$ nonfat milk, $1 \%$ BSA, and $0.3 \%$ Triton X-100 in 0.1 m PBS unless otherwise has been mentioned.

\section{GFAP, CSPGs, and C4S-DS}

The frozen slides were air dried at room temperature for $10 \mathrm{~min}$ and then were washed with PBS for $10 \mathrm{~min}$. The sections were blocked and then incubated with primary antibodies. The following primary antibodies were used overnight at $4^{\circ} \mathrm{C}$ : rabbit anti-GFAP (1:1000, Millipore Bioscience Research Reagents) for astrocytes, mouse anti-CSPGs (CS56, Sigma, 1:200), mouse anti-chondroitin-4-sulfate (C4S, 1:200, Millipore Bioscience Research Reagents) for visualization of CSPG GAG digestion. The slides were washed in PBS three times and then incubated with fluorescent Alexa 568 or 488 or 647 goat-anti mouse secondary antibodies (Invitrogen, 1:400) for $1 \mathrm{~h}$. The slides were coverslipped with Mowiol mounting medium containing DAPI to counterstain the nuclei. The images were taken using a Zeiss 510 laser confocal microscope or Leica epifluorescence microscope.

\section{Neural cell markers}

To assess the antigenic properties of transplanted YFP-NPCs, the frozen slides of transplanted rats were blocked and incubated with the following primary antibodies: mouse anti-nestin (1:200; Millipore Bioscience Research Reagents) for NPCs, rabbit anti-PDGF- $\alpha \mathrm{R}$ (1:40; Santa Cruz Biotechnology) for oligodendrocyte progenitor cells, mouse anti-GFAP (1: 1000; Millipore Bioscience Research Reagents) for astrocytes, mouse anti-adenomatous polyposis coli (APC; 1:50; Calbiochem), mouse antimyelin basic protein (MBP; 1:1000; Sternberger Monoclonal) for mature oligodendrocytes, mouse anti-MAP-2a,b (1:200; Sigma), and mouse anti- $\beta$ tubulin III (1:500; Covance) for neurons, rabbit anti-NF200 (1: 500; Sigma) for axons, and rabbit anti-p75 (1:1000; Millipore Bioscience Research Reagents) and chicken-anti P0 (1:500, Aves Lab) for Schwann cells, mouse anti-OX42 (1:40, Serotec) for macrophages/microglia. The slides were washed in PBS three times and incubated with fluorescent Alexa 568 goat anti-mouse, -rabbit, or -chicken secondary antibody (1: 400; Invitrogen) for $1 \mathrm{~h}$. In double staining for MBP and NF200, the slides were treated with mouse anti-MBP antibody first and then incubated with Alexa 568 secondary antibody. For the second labeling, the slides were incubated with rabbit anti NF200 antibody and subsequently incubated with Alexa 647 secondary antibody (1:400; Invitrogen). The slides were washed and coverslipped with Mowiol mounting medium containing DAPI.

\section{$B D A$ and $P K C-\gamma$}

Four transverse sections (50 $\mu$ m thickness) per animal at 4, 6, 9, and 11 $\mathrm{mm}$ rostral to the injury site were selected for immunohistochemical processing against BDA and protein kinase $\mathrm{C}-\gamma(\mathrm{PKC} \gamma)$. For BDA visualization, free-floating sections were incubated with the following reagents: $0.3 \%$ hydrogen peroxide $\left(\mathrm{H}_{2} \mathrm{O}_{2}\right)$ in absolute methanol for $30 \mathrm{~min}$ at $4^{\circ} \mathrm{C}$ (using blocking solution as above), Vectastain $\mathrm{AB}$ (ABC Elite Kit, Vector Laboratories) in PBS containing $0.5 \%$ Triton X-10 according to manufacturer instructions for $2 \mathrm{~h}, 0.05 \%$ diaminobenzidine, and $0.05 \%$
$\mathrm{H}_{2} \mathrm{O}_{2}$ in PBS for $\sim 5$ min. Sections were mounted onto slides and air dried. Then, the slides were dehydrated through an alcohol series, cleared in xylene, and coverslipped. For PKC $\gamma$ immunostaining, the free-floating sections were blocked and then incubated with anti-PKC $\gamma$ antibody (1: 200, Santa Cruz Biotechnology) overnight. After washes in PBS, the sections were incubated with fluorescent Alexa 568 goat-anti mouse secondary antibody (Invitrogen, 1:400). The sections were slide mounted and coverslipped with Mowiol.

\section{5-HT, synaptophysin, GAP-43, and CGRP}

Free-floating (50 $\mu \mathrm{m}$ thickness) or frozen sections (30 $\mu \mathrm{m}$ thickness) at various distances relative to the injury epicenter (1750, 3000, 5000, 7000, 9000 , and $11,000 \mu \mathrm{m}$ both rostrally and caudally) were processed for double immunohistochemistry for 5-HT/GAP-43, calcitonin generelated peptide (CGRP)/synaptophysin, and CGRP/GAP-43 as described above. Briefly, sections were blocked and then incubated in the following solutions (with three PBS washes after each step): the first primary antibody either anti-rabbit 5-HT (1:10,000, ImmunoStar) or anti-rabbit CGRP (1:1000, Millipore Bioscience Research Reagents) for overnight at $4^{\circ} \mathrm{C}$, Alexa 568 goat anti-rabbit secondary antibody $(1: 400,1 \mathrm{~h})$, second primary antibody either anti-mouse synaptophysin (1:5000, Millipore Bioscience Research Reagents, $2 \mathrm{~h}$ at room temperature) or anti-mouse GAP-43 (1:1000, Millipore Bioscience Research Reagents, $2 \mathrm{~h}$ at room temperature), and then Alexa 647 goat anti-mouse secondary antibody (1:400, $1 \mathrm{~h})$.

\section{C-fos}

Free-floating sections (50 $\mu \mathrm{m}$ thickness) from various distances caudal to the injury center at 6,9 , and $11 \mathrm{~mm}$ were processed for $c$-fos immunostaining. The sections were treated with the following reagents with PBS washes after each step: permeabilized with $0.1 \%$ Triton X-100 in PBS for 5-10 min at room temperature, incubated with $1 \% \mathrm{H}_{2} \mathrm{O}_{2}$ in PBS for 20 min, block with $1 \%$ BSA $+5 \%$ milk and $0.3 \%$ Triton-X in PBS for $1 \mathrm{~h}$, incubated with rabbit anti-c-fos (1:2500, Calbiochem) overnight at $4^{\circ} \mathrm{C}$, incubated with a solution of the HRP conjugate (1:100) for 30-60 min at room temperature, incubated with tyramide labeling solution (Invitrogen) according to the manufacturer's instructions, and then incubated with streptavidin 594 (1:400) for $1 \mathrm{~h}$ at room temperature. The sections were mounted onto slides and coverslipped with Mowiol containing DAPI.

\section{Immunoblotting for CSPGs and GFAP}

Spinal cords were dissected, placed in ice-cold artificial CSF (124 mM $\mathrm{NaCl}, 3 \mathrm{~mm} \mathrm{KCl}, 1 \mathrm{~mm} \mathrm{NaHPO}_{4}, 26 \mathrm{~mm} \mathrm{NaHCO}_{3}, 1.5 \mathrm{~mm} \mathrm{MgSO} 4,1.5$ $\mathrm{mm} \mathrm{CaCl} 2$, and $10 \mathrm{~mm}$ glucose), and cleaned from meninges and nerve roots. One centimeter of the cord centered at the injury site was dissected and homogenized (in a buffer composed of $50 \mathrm{~mm}$ Tris- $\mathrm{HCl}, \mathrm{pH} 7.4,1$ mм EDTA, 0.1\% SDS, $100 \mu \mathrm{m}$ leupeptin, $1 \mu \mathrm{M}$ pepstatin, $10 \mu \mathrm{g} / \mathrm{ml}$ aprotinin, and $100 \mu \mathrm{m}$ phenylmethylsulfonyl fluoride). For slot blotting, $3 \mu \mathrm{g}$ of protein/well was transferred to polyvinylidene difluoride membranes (Millipore) using Bio-Dot slot blot apparatus (Bio-Rad) according to the manufacturer's instructions. The membranes were then incubated with mouse-anti CSPGs (CS-56, 1:300, Sigma) overnight at $4^{\circ} \mathrm{C}$. Following the incubation with HRP-conjugated goat anti-mouse antibody (1:5000), membranes were incubated in ECL plus immunoblotting detection reagents (GE Healthcare) according to the manufacturer's specifications. Immunoreactive bands were quantified using Gel-Pro Analyzer software (Media Cybernetics). To control for equal protein loading, membranes were also probed for actin and CSPG values were normalized to $\beta$-actin (1:200, Millipore Bioscience Research Reagents). Controls for immunoblotting included either a competition assay with incubation of the primary antibody with its target peptide and subsequent incubation on the membranes or loading BSA (bovine serum albumin) as a negative protein control.

\section{Image processing and analysis}

In all neuroanatomical procedures, quantification was executed in an unbiased manner by examiners blinded to the treatment groups based on the previously described methods by our group and others (Karimi- 
Abdolrezaee et al., 2004, 2006; Tang et al., 2004, 2007; Barritt et al., 2006; Biernaskie et al., 2007; Eftekharpour et al., 2007).

\section{Immunohistochemical analysis of CSPGs and GFAP}

For immunodensity measurements of CSPG and GFAP, we selected two mid-horizontal sections per each rat that contained the lesion cavity (100 $\mu \mathrm{m}$ apart). We photographed the entire horizontal section at $10 \times$ primary objective using Tiling and Stitching Software (StereoInvestigator). Then, using NIH Image analysis system, we traced the entire lesional and perilesional areas demarked by the glial scar in each section. Furthermore, we performed automatic thresholding for each image using NIH ImageJ software to determine the threshold for specific signal. After setting the threshold, the immunodensity above the threshold was automatically calculated. Then, we divided the integrated density by the sample area to calculate the mean density per unit area. This calculation was performed to compensate for the different size of the region of interest in the spinal cord.

\section{Analysis of transplant volume, NPC cell survival, and NPC differentiation}

These analyses were performed based on previously described methods by our group and others (Karimi-Abdolrezaee et al., 2006; Biernaskie et al., 2007; Eftekharpour et al., 2007).

Assessment of NPC transplant volume and cell survival was performed on serial spinal cord sections (minimum 200-300 $\mu \mathrm{m}$ apart). We undertook a mathematically unbiased method to determine the volume of NPC transplant in both ChABC-treated/NPC-transplanted and vehicle-treated/NPC-transplanted groups ( $n=6$ /group), we imaged the whole cross sections of the serial spinal cord sections (200-300 $\mu \mathrm{m}$ apart) that contained YFP-NPCs. These images were captured at $10 \times$ primary magnification on a Leica fluorescent microscope using an Image Tiling and Stitching software. Then, using ImageJ Software (NIH), the regions containing YFP-expressing cells were traced on each section and the area was calculated. Then, using the equation $v=\Sigma$ (thickness of section $\times$ number of sections in each sampling block $\times$ area), we calculated the volume of transplant (in cubic millimeters). For quantifying the survival of transplanted YFP-NPCs, we also used an unbiased stereologically based technique. We photographed 10-20 sections (minimum 200-300 $\mu \mathrm{m}$ apart) from each animal containing YFP-NPCs on a confocal microscope with $25 \times$ primary magnification. This distance was used to avoid overcounting of same NPCs. Then, within each spinal cord cross section, we randomly selected up to 10 fields $(368 \times 368 \mu \mathrm{m})$ within the transplant region (depending on the extent of transplant in that spinal cord section). Then, we counted the number of YFP-NPCs containing a nucleus (YFP/DAPI-positive cells) within each given field, and the mean density (in cells per cubic millimeter) for each field was calculated. The total number of surviving YFP-positive cells was then estimated by multiplying the averaged mean density by the transplant volume in each engrafted rat. We only counted the YFP cell bodies that contained normal DAPI stained nucleus to avoid counting the nonviable YFPs or other autofluorescent objects. This criterion was also used to distinguish the thick YFP cell processes that resembled as a cell body and that could cause overestimating the NPC counts. For imaging, we determined the appropriate confocal setting in the first microscopy session that would avoid signal saturation. We then reused the same setting for the subsequent imaging sessions.

To quantify the differentiation pattern of engrafted cells in ChABCand vehicle-treated rats ( $n=6$ /group), we immunostained horizontal $\mu$ sections of the spinal cords containing YFP-positive cells at 9 weeks after transplantation. For each cell marker, we randomly selected three tissue sections that were $200-300 \mu \mathrm{m}$ apart. Using images taken by confocal microscopy at $25 \times$ primary magnification, we first counted the number of YFP/DAPI-positive cells in 10 random fields per section (368 $\mu \mathrm{m} \times 368 \mu \mathrm{m})$. Next, we counted the YFP cells that were colabeled with the cell marker. We only quantified the YFP cell bodies that contained a nucleus (identified with DAPI). Then, we calculated the percentage of colabeled YFP/cell marker for each rat.

\section{Quantification of axonal density and sprouting in the} corticospinal tract

To examine the integrity of main CST, we used immunostaining for PKC $\gamma$ (Mori et al., 1990; Bradbury et al., 2002; Barritt et al., 2006), which specifically marks main CST in the dorsal column, rather than analysis of $\mathrm{BDA}$ anterograde labeling due to interanimal variation in the BDA labeling efficiency. However, since the specificity of PKC- $\gamma$ in detecting CST terminals in gray matter is limited by its diffuse labeling as well as by the expression of PKC- $\gamma$ interneurons (Mori et al., 1990), we used anterograde BDA labeling to study the sprouting of the CST fibers in the spinal gray matter. To correct for interanimal variation in the BDA labeling efficiency, we normalized the intensity value of the BDA-labeled collaterals in the gray matter to the intensity of BDA-labeled fibers in the main CST. For the assessment of PKC- $\gamma$ in the CST, we photographed the dorsal columns of the spinal cord at $10 \times$ primary magnification using a confocal microscope at $4,6,9$, and $11 \mathrm{~mm}$ rostral to injury center $(n=$ 5-6/group). Using ImageJ Software (NIH), we measured the relative density of PKC- $\gamma$ immunoreactivity in a traced area within the dorsal columns (depicted as a box in Fig. 4A). Background intensity from an area with no PKC- $\gamma$-positive immunoreactivity was also subtracted from the intensity value to correct for nonspecific reactions. Furthermore, we performed automatic thresholding for each image using ImageJ software to determine the threshold for specific signal. After setting the threshold, the immunodensity above the threshold was quantified. Then mean net density for PKC- $\gamma$ was calculated by dividing the immunointensity to the traced area. For collateral sprouting in spinal gray matter, we undertook the same approach as described above for PKC- $\gamma$ to measure the relative intensity of BDA labeling within a defined region of the dorsal and intermediate gray matter (see Fig. $4 I_{i}$, boxed region). Then, we normalized the intensity value of the BDA-labeled collaterals in the gray matter to the intensity of BDA-labeled fibers of the main CST to correct for interanimal variation in the BDA labeling efficiency.

\section{Quantification of serotonergic fibers (5-HT)}

To assess 5-HT immunoreactivity, we photographed the cross sections of the entire spinal cord at $10 \times$ primary magnification using on a Leica microscope at eight distances both rostral and caudal to injury center $(1.75,3,5$, and $7 \mathrm{~mm})$. Using Image Software $(\mathrm{NIH})$, we measured the relative density of 5-HT immunoreactivity in entire cross section of the spinal cord (see traced areas in Fig. 5A-F) ( $n=5-6 /$ group). Background intensity from an area with no PKC- $\gamma$-positive immunoreactivity was also subtracted from the intensity value to correct for nonspecific reactions. Furthermore, we performed automatic thresholding for each image using ImageJ software to determine the threshold for specific signal. After setting the threshold, the immunodensity above the threshold was quantified. Then, the 5-HT immunointensity was divided by the spinal cord area to calculate the mean net density/unit area.

\section{Quantification of CGRP and synaptophysin}

To assess CGRP-positive primary afferents terminating in the dorsal horn of the spinal cord, we photographed two dorsal horns for each rat at $10 \times$ primary magnification using a Zeiss confocal microscope at 4,6 , and $9 \mathrm{~mm}$ both rostral and caudal to injury epicenter ( $n=5-6 /$ group). Using ImageJ Software (NIH), we measured the relative density of CGRP immunoreactivity in laminae III-V of two dorsal horns (see Fig. 6A). Then, the CGRP intensity was divided by the traced area to calculate the mean net intensity/unit area. We determined the level of background/ autofluorescence by imaging unstained samples. This reference intensity was then subtracted from the average intensity to determine the net final density. Furthermore, we performed automatic thresholding for each image using NIH ImageJ software to determine the threshold for specific signal. After setting the threshold, the immunodensity above the threshold was quantified. An average of the CGRP intensity from two dorsal horns per section was calculated for each distance in each animal. To examine the density of synaptic terminals made by CGRP primary afferents in the dorsal horn, using ImageJ Software (Co-localization Finder application), we quantified the percentage of colocalization between CGRP and synaptophysin immunoreactivity signals in laminae I-V of dorsal horns (see Fig. 7). 
Quantification of c-fos

For assessment of synaptic connectivity of primary afferents in the dorsal horn, we studied the expression of $c$-fos in the superficial laminae of two dorsal horns of the spinal cord at three distances caudal to the injury center $(4,6$, and $9 \mathrm{~mm})$. For relative comparison of $c$-fos expression across the treatment group, we counted the number of $c$-fos-immunoreactive nuclei in the superficial laminae of two dorsal horns in the transverse sections of the spinal cord at above distances based on a previously described method by Barritt et al. (2006). Two spinal cord cross sections (200-300 $\mu \mathrm{m}$ apart) per each distance per each rat were examined. Then, average of the number of $c$-fos-positive nuclei of the two dorsal horns per section was calculated for each animal at a given distance ( $n=6 /$ group).

\section{Neurobehavioral assessments}

All neurobehavioral assessments were performed and analyzed by at least two examiners "blinded" to treatment groups.

\section{$B B B$ open field locomotor score}

Hindlimb neurobehavioral testing was performed using the Basso, Beattie, Bresnahan Locomotor Rating Scale (Basso et al., 1995). For this evaluation, the rats were placed individually in an open field with a nonslippery surface. The 22-point (0-21) BBB scale was used to assess hindlimb locomotor recovery including joint movements, stepping ability, coordination, and trunk stability. A score of 21 indicates unimpaired locomotion as observed in uninjured rats. The tests were performed by two examiners who were blinded to the animals' treatments. The duration of each session was 4 min per rat. BBB tests were performed weekly after injury and after treatments for 8 weeks after transplantation (15 weeks after injury). BBB scores generated on week 5 were used to block randomize chronically injured rats into five experimental groups $(n=$ 13-15 rats per group) (see Table 1 for details). We randomly allocated the injured animals into five experimental groups in the manner that all groups consisted of animals with comparable range of BBB scores as well as group average. This randomization ensured the presence of equivalent locomotor deficits across the groups before the commencement of treatments.

\section{Grid-walking analysis}

We performed grid walk analysis to assess deficits in fine motor functions ( $n=13-15$ rats/group). The rats were allowed to cross a 10-cm-wide and 1 -m-long horizontal runway of wooden grids, which were elevated $30 \mathrm{~cm}$ from the ground. To avoid habituation of animals to the fixed bar spacing, the bars were irregularly spaced. Animals were trained for three sessions before injury. For baseline, postinjury, and posttreatment tests, each animal walked on the grid three times. The tests were recorded on digital videotape, and analyses were done offline in slow motion in a blinded manner. For quantitative purposes, we counted the number of hindlimb footfalls that occurred within an identified $75 \mathrm{~cm}$ length of the crossway. One recording was performed before injury to determine the baseline for each animal. After injury, due to the loss of weight support, recordings were started 3 weeks after injury when the injured rats began to show adequate weight support and then, the analyses were performed biweekly thereafter up to 15 weeks after injury. No recording was done between weeks 6 and 8 due to consecutive treatment surgeries on the rats. Assessments were resumed at week 9. For each session, the average number of complete hindpaw falls of each rat was taken from two to three trials. In the baseline (normal) measurements, no stepping errors were observed. The injured rats with dragging hindlimbs were scored the maximum footfalls of 15 .

\section{Assessment of mechanical and thermal allodynia}

All rats were also tested for mechanical and thermal allodynia on a biweekly basis after injury and treatments starting 3 weeks after SCI based on previously established methods for the assessments of neuropathic pain after SCI (Bruce et al., 2002; Gris et al., 2004; Oatway et al., 2004, 2005; Fleming et al., 2008). In brief, mechanical allodynia was evaluated by 10 mechanical stimuli ( $2 \mathrm{~g}$ von Frey filament) to the dorsal trunk area rostral, around, and caudal to the lesion site (areas corresponding to the fifth through ninth thoracic spinal segments). Each stimulus lasted $3 \mathrm{~s}$ and was separated by a $5 \mathrm{~s}$ interval period. The number of avoidance responses (trunk shakes, jumping, escaping, vocalization, or aversive reactions) were counted and recorded. Thermal allodynia was assessed by tail flick in response to a light beam that was applied to the tail. The latency of the rat to remove its tail from the heat was recorded. This latency was used as a measure to indicate thermal allodynia.

\section{Statistical analysis}

Statistical analyses for neurobehavioral data were performed using SigmaStat software (SPSS) by two-way repeated-measures ANOVA comparing groups versus time points followed by post hoc pairwise multiple comparison using the Student-Newman-Keuls method. Differences in the observed frequency of consistent stepping between all groups in BBB assessment were examined using the $\chi^{2}$ test. For neuroanatomical studies, statistical analyses of intensity measurements and cell counts were tested by one-way ANOVA comparing groups followed by post hoc pairwise multiple-comparison testing by the Holm-Sidak method or by Student's $t$ tests where two groups were compared. Data are reported as means \pm SEMs, and $p<0.05$ was considered significant.

\section{Results}

\section{The effects of CSPGs on NPC survival in the chronically injured spinal cord}

We previously reported that in vivo delivery of EGF, bFGF, and PDGF-AA greatly optimized the survival of engrafted adult NPCs in subacute ( 2 weeks after lesion) SCI (Karimi-Abdolrezaee et al., 2006). However, this strategy failed to exert similar effects after chronic transplantation 6 weeks following traumatic injury. While $\sim 37 \%$ of NPCs survived and integrated in subacutely injured spinal cord, in a parallel study we found a marked decrease in the long-term survival of YFP-NPCs after chronic transplantation (Karimi-Abdolrezaee et al., 2006). At 8 weeks after NPC transplantation in the chronically injured spinal cord, clusters of nonviable YFP profiles that had failed to disperse and integrate within the host spinal cord were observed around the transplantation sites (Fig. $1 A-C$ ). Interestingly, immunohistochemical analysis revealed intense CSPG immunoreactivity associated with astrocytes surrounding the nonviable YFP profiles (Fig. $1 C, D-G)$, suggesting that CSPGs associated with the glial scar might be involved in mediating the suboptimal survival of transplanted NPCs in chronic lesions.

\section{Degradation of CSPGs by ChABC treatment in chronic SCI}

To examine the potential contribution of CSPGs in mediating the suboptimal outcomes of NPC transplantation after chronic SCI, we disrupted CSPGs in the chronically injured spinal cord with ChABC. Targeting CSPGs by the intrathecal delivery of ChABC at 6 weeks after injury for 1 week resulted in a pronounced reduction in CSPGs in the perilesional areas compared to the vehicle treatment (Fig. 1H,I). Successful enzymatic degradation of CSPGs was further confirmed by immunohistochemistry against $\mathrm{C} 4 \mathrm{~S}$, which marks the tetrasaccharide linker region, or stub, that results from $\mathrm{ChABC}$ enzymatic action. While no apparent endogenous C4S immunoreactivity was detected in uninjured or nonChABC-treated injured animals (Fig. $1 \mathrm{~J}$ ), rats treated with ChABC exhibited a pronounced expression of C4S in the spinal cord tissue surrounding the lesion site (Fig. $1 K, L$ ). Using two complementary quantitative approaches, slot blot and immunohistochemistry analyses for anti-chondroitin sulfate CS56 antibody that recognizes the intact GAG side chains of CSPGs, we found significant reduction in expression of CSPGs in ChABC-treated chronically injured rats $(n=5)$ compared to the vehicle-treated counterparts $(n=4)$ (Fig. $1 I, O)$. While ChABC treatment did not entirely remove CSPGs, slot blot analysis re- 

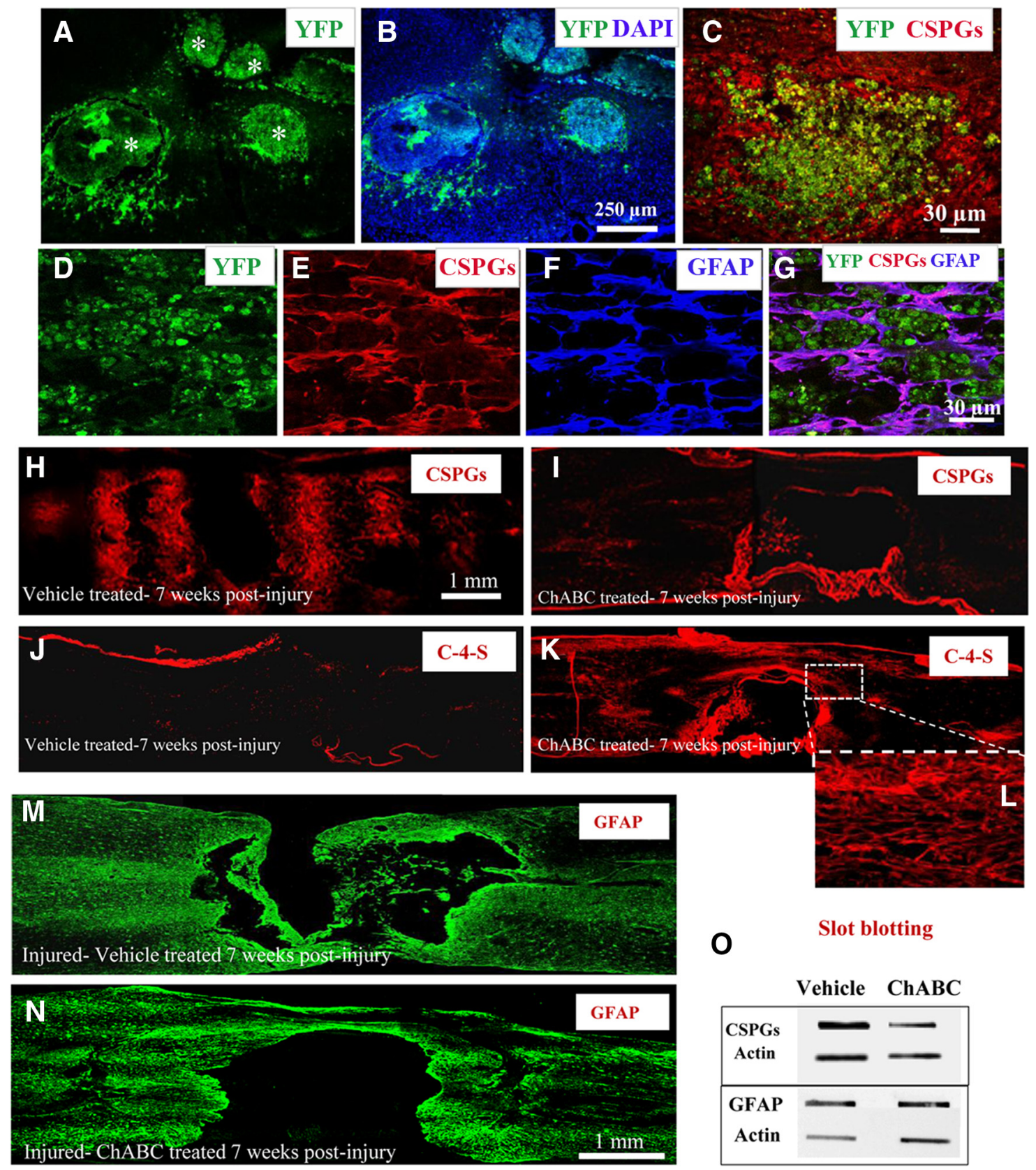

Figure 1. CSPGs restrict transplantation of NPCs in chronic spinal cord injury. $A, B$, Engrafted YFP-positive NPCs (green) appear in clusters after chronic transplantation. At 6 weeks after transplantation, the majority of engrafted NPCs appear as clusters of nonviable YFP-positive profiles ( ${ }^{*}$ ) surrounded by some surviving NPCs. C, Confocal immunostaining revealed dense deposits of CSPGs (red) around the nonviable YFP cells (green). D-G, Immunofluorescence labeling demonstrated the association of CSPGs (red) with astrocytes (blue) that surround the nonviable YFP-NPCtransplanted cells (green). $\boldsymbol{H}-\mathbf{L}, \mathbf{O}$, Treatment with ChABC leads to significant degradation of CSPGs in chronic SCI. $\boldsymbol{H}$, Confocal image of a longitudinal section of vehicle-treated spinal cords 7 weeks after SCI shows the intense expression of CSPGS (red) around the injury center. $I$, Treatment with ChABC at 6 weeks after injury for 1 week substantially reduced CSPG deposits in the chronically injured spinal cord. J-L, Successful degradation of CSPGs was further confirmed by immunohistochemistry against C4S, which marks the tetrasaccharide linker region, or stub, that results from ChABC enzymatic action. $\boldsymbol{J}, \boldsymbol{K}$, While vehicle-treated rats showed no $(4 S$ immunoreactivity in their spinal cord $(\boldsymbol{J})$, rats that received $C h A B C$ treatment showed $(4 S$ immunoreactivity in their spinal cord $(\boldsymbol{K})$. Inset image in $\boldsymbol{L}$ represents the enlarged box in $\boldsymbol{K}$ that shows a magnified area of C4S immunoreactivity in ChABC-treated chronically injured rats. $\boldsymbol{O}$, Significant degradation of CSPGs by ChABC was quantified by slot blotting against CS56 antibody, which showed a 4.5 -fold decrease in the expression of CSPGs in the ChABC-treated group (21.96 $\pm 2.31 \%$ ) compared to the vehicle-treated group $(100 \pm 30 \%, p<0.01$, Student's $t$ test). Analysis of CSPG immunointensity on the chronically injured spinal cord sections around the SCI lesion also confirmed a 2.5-fold decrease in CSPGs after ChABC treatment $(39.5 \pm 2.66 \%)$ compared to the vehicle-treated group $(100 \pm 11 \%, p<0.01$, Student's $t$ test. $M$, $N$, Confocal images of vehicle- and ChABC-treated rats showing the expression of GFAP in the glial scar around the lesion area. Quantitative immunohistochemistry analysis showed no significant differences in the expression of GFAP in the spinal cords of the ChABC- (127.27 \pm 7.3) and vehicle- $(100 \pm 11.99)$ treated rats around the lesional and perilesional areas $(p>0.05$, Student's $t$ test). $\boldsymbol{0}$, This result was also confirmed by quantitative slot blot analysis, which revealed no significant difference in the expression of GFAP between the vehicle-treated (100 $\pm 19 \%)$ and the ChABC-treated (116 $\pm 12.21 \%)$ groups around the SCI lesion site ( $p>0.05$, Student's $t$ test).

vealed a 4.5 -fold reduction in CSPG expression in the ChABCtreated group $(21.96 \pm 2.31 \%)$ compared to the vehicle-treated group $\left(100 \pm 30 \%,{ }^{* *} p<0.01\right.$, Student's $t$ test $)$ (Fig. 1O). Analysis of CSPG immunointensity on the chronically injured spinal cord sections also confirmed a 2.5-fold decrease in CSPGs after ChABC treatment $(39.5 \pm 2.66 \%)$ compared to the vehicletreated group $(100 \pm 11 \%, p<0.01$, Student's $t$ test) (Fig. 1O). Furthermore, we examined whether the ChABC treatment also 

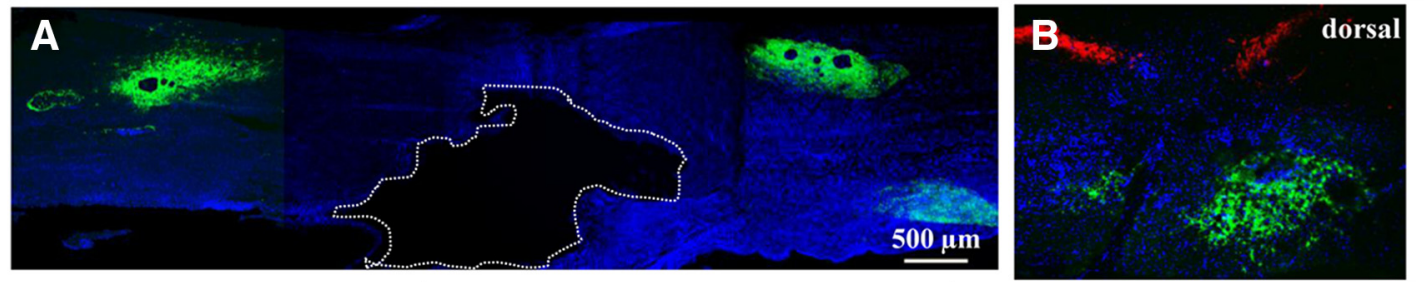

\section{NPC/DAPI- ChABC treated}

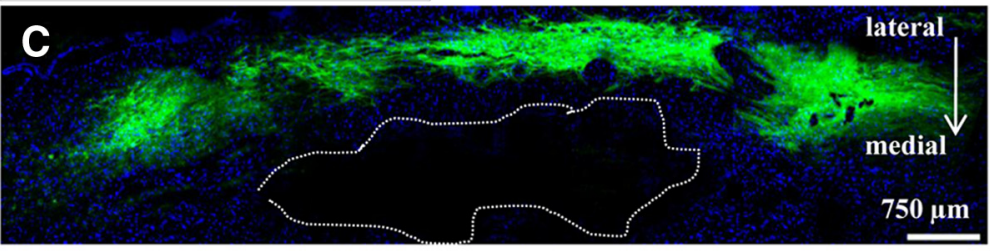

NPC/CGRP/DAPI- ChABC treated
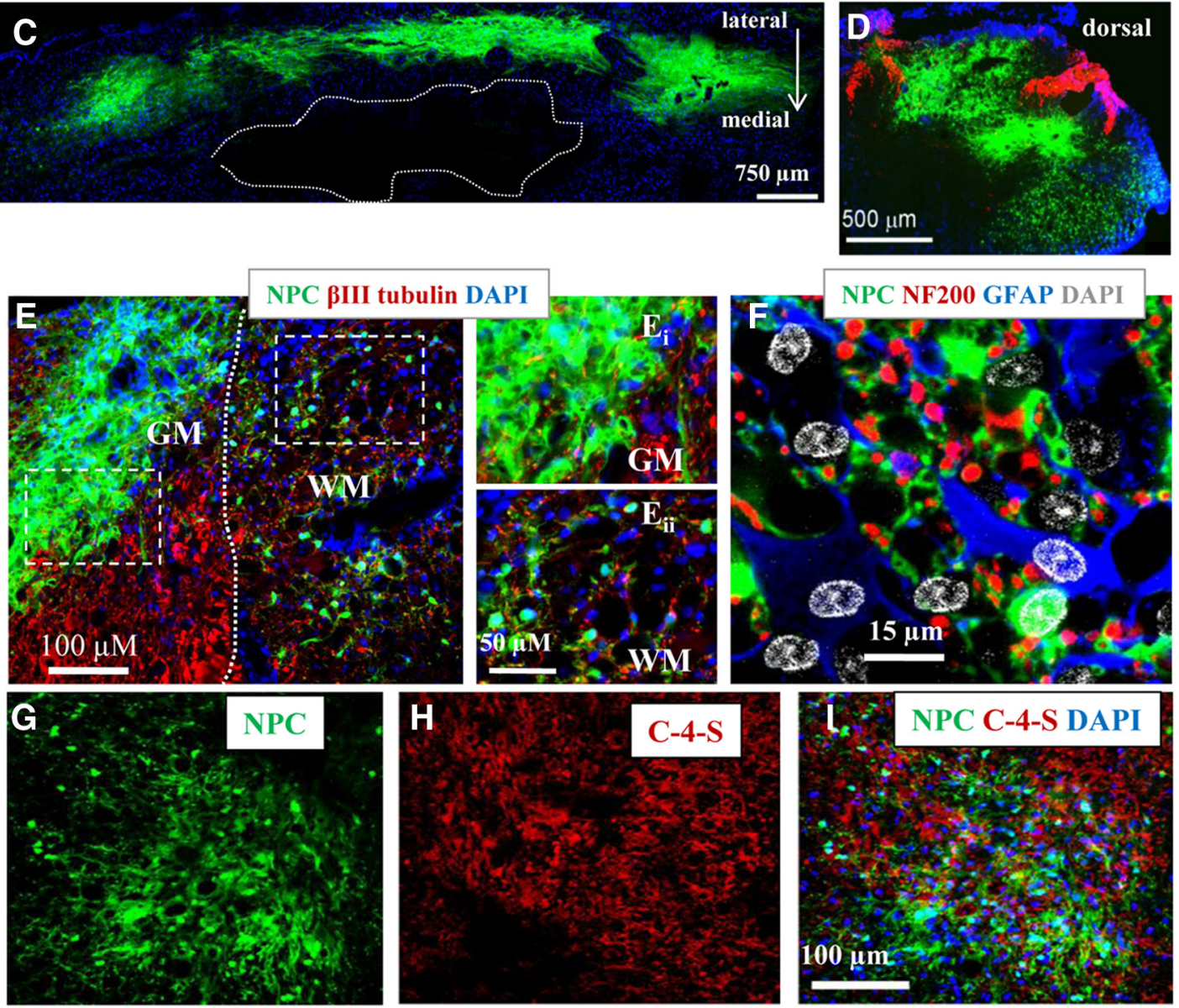

Figure 2. ChABC treatment greatly optimizes NPC transplantation in chronically injured spinal cord. $A-D$, Confocal images of longitudinal and cross sections of chronically injured spinal cord transplanted with NPCs at 9 weeks after transplantation show a significant increase in the number of surviving NPCs ( $g r e e n)$ in ChABC-treated spinal cord $(\boldsymbol{C}, \boldsymbol{D})$ compared to the vehicle-treated spinal cords $(\boldsymbol{A}, \boldsymbol{B})$. D , The majority of NPCs integrated within white matter areas including dorsal, lateral, and ventral columns. Quantification of transplant volume revealed a 6.2 -fold increase in ChABC-treated rats $\left(n=6,3.7 \pm 0.79 \mathrm{~mm}^{3}\right)$ compared to vehicle-treated rats $\left(n=6,0.6 \pm 0.24 \mathrm{~mm}^{3}\right)(p<0.01$, Student's $t$ test). NPC cell survival analysis also showed a 5.7 -fold increase in number of surviving NPCs (YFP/DAPI-positive cells) in ChABC-treated rats (28.25 $\pm 6.77 \%)$ relative to the vehicle-treated ones $(4.9 \pm 1.96 \%)(p<0.01$, Student's $t$ test). ChABC treatment resulted in a significant increase in rostral and caudal migration of NPCs from the injection sites $(4.2 \pm 0.50 \mathrm{~mm})$ compared to vehicle treatment $(1.4 \pm 0.4 \mathrm{~mm})(p<0.001$, Student's $t$ test). We also found a 4.6 -fold increase in the total length of rostrocaudal distribution of NPCs in ChABC-treated rats $(13.02 \mathrm{~mm}$ in the best case, average $8.32 \pm 1.5 \mathrm{~mm})$ compared to vehicle-treated rats ( $2.24 \mathrm{~mm}$ in the best case, average $1.82 \pm 0.5 \mathrm{~mm})\left(p<0.005\right.$, Student's $t$ test). $\boldsymbol{E}$, Integrated NPCs localized in gray matter $\left(\boldsymbol{E}_{i}\right)$ showed a greater tendency to form clusters, whereas NPC sin white matter regions $\left(\boldsymbol{E}_{i j}\right)$ were dispersed with extending projections. $\boldsymbol{F}, \mathrm{NPC}$ (green) in white matter regions ensheathed the injured axons (NF200, red) and showed a normal relationship with host astrocytes (GFAP, blue). G-I, Confocal images depict C4S immunostaining marking the degraded products of CSPGs in chronically injured spinal cord 10 weeks after ChABC treatment. Integrated NPCs around the lesion site were localized within or very close to the areas with C4S immunoreactivity, confirming the impact of inhibitory influence of CSPGs on the survival and integration of engrafted NPCs.

affected the population of reactive astrocytes in the glial scar around the SCI lesion. Using slot blot and immunohistochemical analyses, we examined the expression of GFAP as a marker for reactive astrocytes (Fig. 1M-O). We focused on the lesional and perilesional areas. Quantitative slot blot analysis showed no significant difference in the expression of GFAP between the vehicle-treated $(100 \pm 19 \%)$ and ChABC-treated (116 \pm
$12.21 \%)$ groups around the SCI lesion site $(p>0.05$, Student's $t$ test) (Fig. 1O). Analysis of GFAP immunointensity on the chronically injured spinal cord sections also confirmed no significant changes in the expression of GFAP between vehicle-treated $(100 \pm 11.99)$ and ChABC-treated (127.27 \pm 7.3) groups around the lesional and perilesional areas $(p>$ 0.05 , Student's $t$ test) (Fig. $1 M, N$ ). 
Table 2. Summary of NPC-transplantation data

\begin{tabular}{|c|c|c|c|c|}
\hline & $\begin{array}{l}\text { NPC cell survival } \\
\text { (cell number and \%) }\end{array}$ & $\begin{array}{l}\text { Transplant volume } \\
\left(\mathrm{mm}^{3}\right)\end{array}$ & $\begin{array}{l}\text { Migration from the } \\
\text { injection sites (mm) }\end{array}$ & $\begin{array}{l}\text { Total rostrocaudal } \\
\text { distribution length }(\mathrm{mm})\end{array}$ \\
\hline \multicolumn{5}{|c|}{ Vehicle-treated/ NPC transplanted } \\
\hline Rat \#1 & $7583(1.9 \%)$ & 0.118 & 0.708 & 1.34 \\
\hline Rat \#2 & $18,518(4.6 \%)$ & 0.68 & 2.1 & 2.18 \\
\hline Rat \#3 & $50,777(12.7 \%)$ & 1.5 & 2.2 & 2.24 \\
\hline Rat \#4 & $10,410(2.6 \%)$ & 0.23 & 1.9 & 2.03 \\
\hline Rat \#5 & $16,453(4.1 \%)$ & 0.34 & 1.1 & 1.99 \\
\hline Rat \#6 & $14,259(3.6 \%)$ & 0.62 & 0.65 & 1.12 \\
\hline Average & $19,667(4.91 \%)$ & 0.58 & 1.47 & 1.82 \\
\hline \multicolumn{5}{|c|}{ ChABC-treated/NPC transplanted } \\
\hline Rat \#1 & $201,506(50.4 \%)$ & 6.04 & 5.99 & 13.02 \\
\hline Rat \#2 & $36,366(9.1 \%)$ & 2.15 & 4.3 & 5.41 \\
\hline Rat \#3 & $84,854(21.2 \%)$ & 1.61 & 3.65 & 7.37 \\
\hline Rat \#4 & $118,888(29.7 \%)$ & 3.21 & 3.86 & 5.38 \\
\hline Rat \#5 & $99,254(31.4 \%)$ & 3.06 & 2.98 & 7.85 \\
\hline Rat \#6 & $125,698(27.8 \%)$ & 5.71 & 4.58 & 10.90 \\
\hline Average & $111,094(28.27 \%)$ & 3.6 & 4.23 & 8.32 \\
\hline
\end{tabular}

\section{The effects of $\mathrm{ChABC}$ treatment on long-term survival of NPCs in chronic SCI}

To examine the effects of CSPG degradation on NPC transplantation in chronic SCI, 1 week after ChABC or vehicle treatment (7 weeks after injury) (see Table 1 for details on experimental paradigms and groups), we treated the same chronically injured rats with NPCs and GFs using approaches previously described by our group (Karimi-Abdolrezaee et al., 2006; Eftekharpour et al., 2007). At 3, 6, and 9 weeks after transplantation, we found a striking increase in the number of surviving YFP-NPCs in the ChABC-treated group (Fig. 2C,D, data shown for 9 weeks) compared to the vehicle-treated group (Fig. 2A,B). While all chronically transplanted rats in both ChABC-treated and vehicle-treated groups showed evidence of NPC survival in their spinal cord at 9 weeks after transplantation, the number of surviving NPCs was remarkably higher in the ChABC-treated group (Fig. $2 C, D)$. Our cell quantification analysis $(n=12,6$ rats/group) at the last experimental time point (9 weeks after transplantation) showed a 5.7-fold increase in the number of surviving NPCs (YFP/DAPI-positive cells) in the ChABC-treated group $(28.27 \pm 6.7 \%$ cell survival, an average of $111,094 \pm 27,259$ YFP/DAPI-positive cells per spinal cord) compared to the vehicle-treated group $(4.91 \pm 1.96 \%$ cell survival, an average of $19,667 \pm 7875$ YFP/DAPI-positive cells per spinal cord, $p<0.01$, Student's $t$ test) (Table 2). The NPC transplant volume also showed a 6.2-fold increase in the ChABC-treated group (3.63 \pm $\left.0.79 \mathrm{~mm}^{3}\right)$ compared to the vehicle-treated group $(0.58 \pm 0.24$ $\mathrm{mm}^{3}, p<0.01$, Student's $t$ test) (Table 2). Similar to our previous studies, which examined the effect of transplantation of NPCs after subacute SCI (Karimi-Abdolrezaee et al., 2006), the transplanted NPCs mainly integrated into and dispersed along the white matter tracts (Fig. $2 \mathrm{~B}$ ). Examination of transverse spinal cord sections revealed that the NPCs had integrated and dispersed in dorsal, lateral, and ventral white matter regions and had also extended processes to ensheath the chronically injured axons (Fig. 2D,E, $\left.E_{i i}, F\right)$. In white matter, the NPC-derived cells also assumed a normal relationship with the neighboring host astrocytes that demonstrated the phenotypic characteristics of normal astrocytes (Fig. 2 F). A smaller percentage of NPCs were found in the spinal cord gray matter or around the legion cavity, and these NPCs mainly appeared as dense cellular clusters. Pretreatment with $\mathrm{ChABC}$ also resulted in significantly enhanced rostral and caudal migration of NPCs from the injection sites $(4.23 \pm 0.50$ $\mathrm{mm})$ compared to vehicle treatment $(1.47 \pm 0.4 \mathrm{~mm}, p<0.01$, Student's $t$ test) (Table 2 ). The enhanced cell migration resulted in improved cell dispersion and therefore a decrease in YFP cell clustering in the ChABC-treated group. Overall, we found a 4.57fold increase in the total length of rostrocaudal distribution of NPCs in ChABC-treated rats $(13.02 \mathrm{~mm}$ in the best case, average $8.32 \pm 1.5 \mathrm{~mm}$ ) (see Table 2) compared to vehicle-treated rats $(2.24 \mathrm{~mm}$ in the best case, average $1.82 \pm 0.5 \mathrm{~mm}, p<0.005$, Student's $t$ test) (Table 2). The transplanted NPCs in ChABC group had also migrated toward the injury site and occupied the entire spared rim of white matter tissue around the lesion (Fig. 2C).

\section{Transplanted NPCs principally reside in areas of CSPG degradation}

To further examine the impact of ChABC degradation on NPC survival in the chronically injured spinal cord, we performed immunohistochemistry against the CSPG breakdown product C4S. Our results demonstrated the long-lasting presence of detectable C4S immunoreactivity at 10 weeks after ChABC treatment (15 weeks after injury) (Fig. $2 H$ ). Furthermore, in the perilesional areas the integrated NPCs were localized within or very close to the areas with C4S immunoreactivity (Fig. 2G-I), providing further evidence in support of the impact of CSPG degradation on promoting the survival and integration of engrafted NPCs.

\section{Transplanted NPCs mainly differentiate along an oligodendroglial lineage}

As we previously reported (Karimi-Abdolrezaee et al., 2006; Eftekharpour et al., 2007), we combined NPCs and the in vivo delivery of growth factors EGF, bFGF, and PDGF-AA at the time of NPC transplantation to promote oligodendroglial differentiation of the transplanted NPCs. Here, we asked whether ChABC treatment alters the outcome of in vivo GF delivery to drive the preferential oligodendroglial differentiation of NPCs in the chronically injured spinal cord. Using cellular markers for different neural lineages, we investigated the differentiation pattern of NPCs in both ChABC-treated and vehicle-treated groups at 9 weeks after transplantation in chronic SCI $(n=5$ for ChABC group and $n=6$ for vehicle group) (Fig. 3). Our findings demonstrated that the majority of transplanted YFP-NPCs differentiated along an oligodendroglial lineage as evidenced by olig2 expression in both groups $(\sim 73 \%$ in the ChABC-treated group 

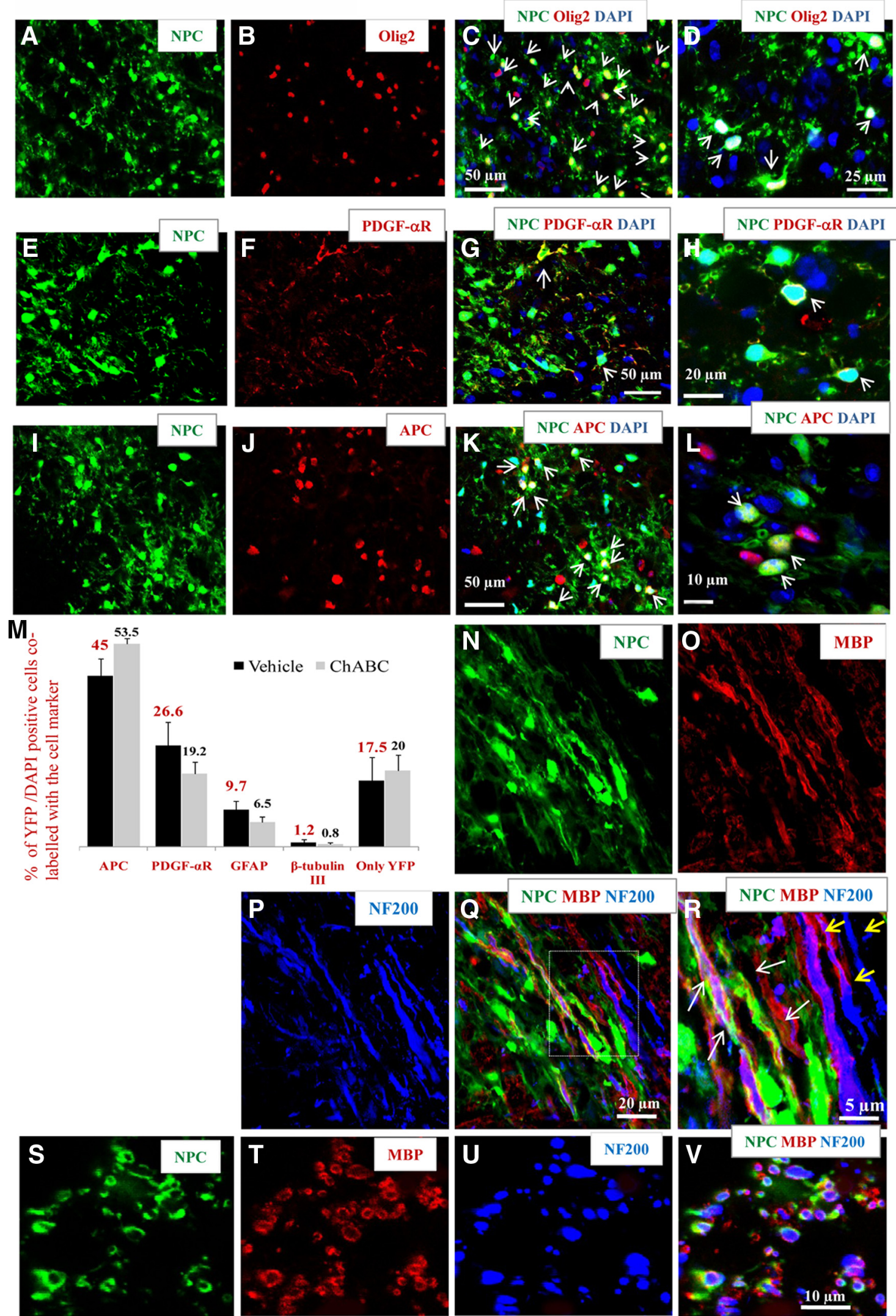

Figure 3. Transplanted adult NPCs principally differentiate into mature oligodendrocytes and remyelinate the chronically injured spinal cord. $A-D$, Confocal images of ChABC/GF $+N P C$-treated spinal cord at 9 weeks after chronic transplantation demonstrate differentiation of NPCs along an oligodendroglial lineage identified by their 0 lig-2 expression (arrows). $\boldsymbol{E}-\boldsymbol{L}$, , These oligodendrocytes were a mixed population of immature (PDGF- $\alpha$ R-positive, arrows) and mature (APC-positive, arrows) cells. $\boldsymbol{M}$, Cell quantification showed that the majority of engrafted NPCs showed an oligodendroglial fate identified by 0 lig2 expression (73\% in the ChABC-treated group and $71 \%$ in the vehicle-treated group). In ChABC groups, $\sim 53.5 \pm 1.5 \%$ and $18.2 \pm 3.2 \%$ of YFP-expressing cells were mature (APC) and immature (PDGF- $\alpha \mathrm{R}$ ) oligodendrocytes. In the vehicle-treated group, $45 \pm 4.6 \%$ and $26.6 \pm 6.3 \%$ of YFP-expressing cells were mature and immature oligodendrocytes, respectively. Only $6.5 \pm 1.4 \%$ and $9.7 \pm 2.47 \%$ generated GFAP-positive astrocytes in the ChABC- and vehicle-treated groups, respectively. Rare neuronal (Figure legend continues.) 
and $71 \%$ in the vehicle-treated group) (Fig. $3 A-D$ ). Furthermore, using antibodies against PDGF- $\alpha \mathrm{R}$ and APC, we identified and quantified $19.2 \pm 3.2 \%$ and $53.5 \pm 1.4 \%$ of YFP cells that showed the phenotypic characteristics of immature and mature oligodendrocytes, respectively, in the ChABC-treated rats and $26.6 \pm 6.3$ and $45.05 \pm 4.6 \%$ in the vehicle-treated group (Fig. $3 E-M$ ). Using confocal imaging and $z$-stack examination, $6.5 \pm 1.42 \%$ of the transplanted NPC were identified as GFAP-positive astrocytes in the ChABC-treated group and $9.7 \pm 2.45 \%$ in the vehicle-treated group (Fig. $3 M$ ). We found only a minimal number of $\beta$-III tubulin-positive neurons $(0.8 \pm 0.3 \%$ in ChABC group and $1.2 \pm 0.8 \%$ in the vehicle group) (Fig. $3 M$, images not shown). Similar to our previous work (Karimi-Abdolrezaee et al., 2006; Eftekharpour et al., 2007), our analysis revealed a population of YFP cells that were not labeled by the neural cell markers that we used $(20 \pm 4.27 \%$ in the ChABC-treated group and $17.5 \pm 6 \%$ in the vehicle group). To further determine the fate of this population, we also used markers for undifferentiated NPCs (nestin), macrophages/microglia (OX42), and Schwann cells (p75 and P0) (images not shown). However, in agreement with our previous report (Karimi-Abdolrezaee et al., 2006), we found no evidence of undifferentiated NPCs (i.e., nestin-positive YFP cells) at 9 weeks after transplantation. We also found no evidence of Schwann cells or macrophages/microglia differentiation among the engrafted NPCs. These results confirm our previous findings that our transplantation strategy directs the fate of adult NPCs toward an oligodendroglial lineage and that combining ChABC treatment remarkably promotes the survival and migration of NPCs without influencing their differentiation in the chronically injured spinal cord.

\section{NPC-derived oligodendrocytes ensheath and remyelinate chronically injured axons}

In previous work, we demonstrated the capacity of NPC-derived oligodendrocytes to ensheath, remyelinate, and improve the molecular and functional properties of spinal cord axons in models of subacute traumatic SCI (Karimi-Abdolrezaee et al., 2006) or congenital dysmyelination (Eftekharpour et al., 2007). Here, we also confirmed the ability of NPC-derived oligodendrocytes to remyelinate chronically injured axons (Fig. $3 N-V$ ). Since YFP expression in the transplanted NPCs is also detectable in the fine cellular processes, we were able to examine remyelination using confocal microscopy and $z$-stack images. Using these techniques, we consistently observed in transplanted animals the MBP-positive processes of oligodendrocytes derived from YFP-positive NPCs that had ensheathed the chronically injured NF200-positive axons (Figs. $2 F$, $3 N-V)$. In the vicinity of these NPC-derived oligodendrocytes, we also found some partially remyelinated axons that were not associated with NPCs, suggesting remyelination by host oligodendrocytes and/or Schwann cells (Fig. 3R).

\section{$\leftarrow$}

(Figure legend continued.) differentiation was also observed $(0.8 \pm 0.5 \%$ in the ChABC group and $1.2 \pm 0.8 \%$ in the vehicle group). At 9 weeks after transplantation, NPCs did not show immunoreactivity for nestin, OX42 (macrophages/microglia), or Schwann cells (P0 and P75) (data not shown). Approximately $20 \pm 4.2 \%$ and $17.5 \pm 6 \%$ of YFP cells in the ChABC and vehicle groups, respectively, were not colabeled with the markers that we used. NPC-mediated remyelination of chronically injured axons is depicted in longitudinal $(\boldsymbol{N}-\boldsymbol{R})$ and transverse $(\boldsymbol{S}-\boldsymbol{V})$ sections. Processes of NPC-derived oligodendrocytes (YFP, green) express MBP (red) and efficiently ensheath the majority of the injured axons (NF200, blue) in their vicinity. $\boldsymbol{R}$, The magnified area in the box in $\mathbf{Q}$ shows the NPC-derived oligodendrocytes that remyelinate the injured axons (white arrows). There are also some partially remyelinated axons by the endogenous cells as well as some unmyelinated axons (yellow arrows).

\section{ChABC and NPC transplantation promote axonal preservation and sprouting of the CST axons after chronic SCI}

To investigate whether the combination of ChABC, GF, and NPC treatments could also promote axonal sparing and plasticity in the chronically injured spinal cord, we next examined the integrity and plasticity of axons in the descending CST, which is significantly disrupted in our model of compressive SCI. CST axons caudal to the injury undergo Wallerian degeneration, whereas the axons in the rostral segment are subjected to retrograde axonal degeneration or "die-back." We assessed the integrity of the main CST using immunostaining for PKC $\gamma$ (Mori et al., 1990; Barritt et al., 2006), which specifically marks the main CST in the base of the dorsal columns of uninjured rats (Fig. 4A,B). At 16 weeks after injury, we found no detectable PKC- $\gamma$ in the dorsal columns at any distances caudal to the injury center, confirming Wallerian degeneration in the caudal CST. PKC- $\gamma$ intensity was also dramatically decreased in the base of the dorsal column at all examined rostral distances (between 4000 and 11,000 $\mu \mathrm{m}$ ) compared to the uninjured spinal cord, suggesting axonal degeneration in rostral CST (Fig. 4). Injured CST fibers frequently appeared as swollen structures indicating dystrophic terminals. While all injured groups showed a significant reduction in the PKC- $\gamma$ immunointensity at points rostral to the lesion in comparison to the uninjured animals (Fig. 4); this reduction was more pronounced in the plain injured group (SCI baseline) than in our four treatment groups (ChABC, GFs, NPCs+GFs, and ChABC/GFs + NPCs) (Fig. 4). After SCI (in all experimental injured groups), PKC- $\gamma$ immunoreactivity was also detected in aberrant regions of the dorsal columns (i.e., areas outside the main CST). Our quantification demonstrated a significantly higher intensity in the staining of PKC- $\gamma$ in the normal areas of the main CST in the combined treatment group (ChABC/GFs + NPCs) than in the plain injured, ChABC, and GF groups at 4000 and $9000 \mu \mathrm{m}$ (Fig. $4 N)(p<0.001$, one-way ANOVA, Holm-Sidak post hoc $)$ and to the plain injured group at $6000 \mu \mathrm{m}$ (Fig. $4 N)(p<0.01$, one-way ANOVA, Holm-Sidak post hoc) rostral to the lesion. While the ChABC, GF, and NPC+GF treatment alone demonstrated a trend of positive effects on the increased intensity of PKC- $\gamma$ in the main CST, their individual effects did not reach a significant level, except for the GF+NPC group, which showed a significant difference compared to the plain injured group at $9000 \mu \mathrm{m}$ rostral to the lesion ( $p<0.05$, one-way ANOVA, Holm-Sidak post hoc) (Fig. $4 N$ ).

Next, we examined the plasticity of CST collaterals innervating the spinal cord gray matter using anterograde BDA labeling. To correct for interanimal variations in the BDA labeling efficiency, we normalized the intensity of the BDA-labeled collaterals in the gray matter to the intensity of BDA-labeled main CST in the same section. Analysis of the dorsal and intermediate gray matter regions (Fig. 4I, boxed area) revealed a significant increase in the density of BDA-labeled collaterals in the ChABC/ $\mathrm{GF}+\mathrm{NPC}$ treatment group when compared to the uninjured group at all distances (ranging from 4000 to $11,000 \mu \mathrm{m}$ ) rostral to the lesion with the highest value detected at $4000 \mu \mathrm{m}$ (a 2.75-fold increase) (Fig. 4O). Furthermore, the density of BDA-labeled collaterals in ChABC/GF+NPC group was significantly higher than all other groups at 4000 and $11000 \mu \mathrm{m}$ and to the plain injured and GF-only groups at 6000 and $9000 \mu \mathrm{m}$ rostral to the lesion (Fig. 4O) ${ }^{* *} p<0.001,{ }^{\star} p<0.01$, one-way ANOVA, Holm-Sidak post hoc). While the ChABC and NPC+GF treatments when administered in isolation also demonstrated a trend toward a positive effect on promoting collateral sprouting of the 

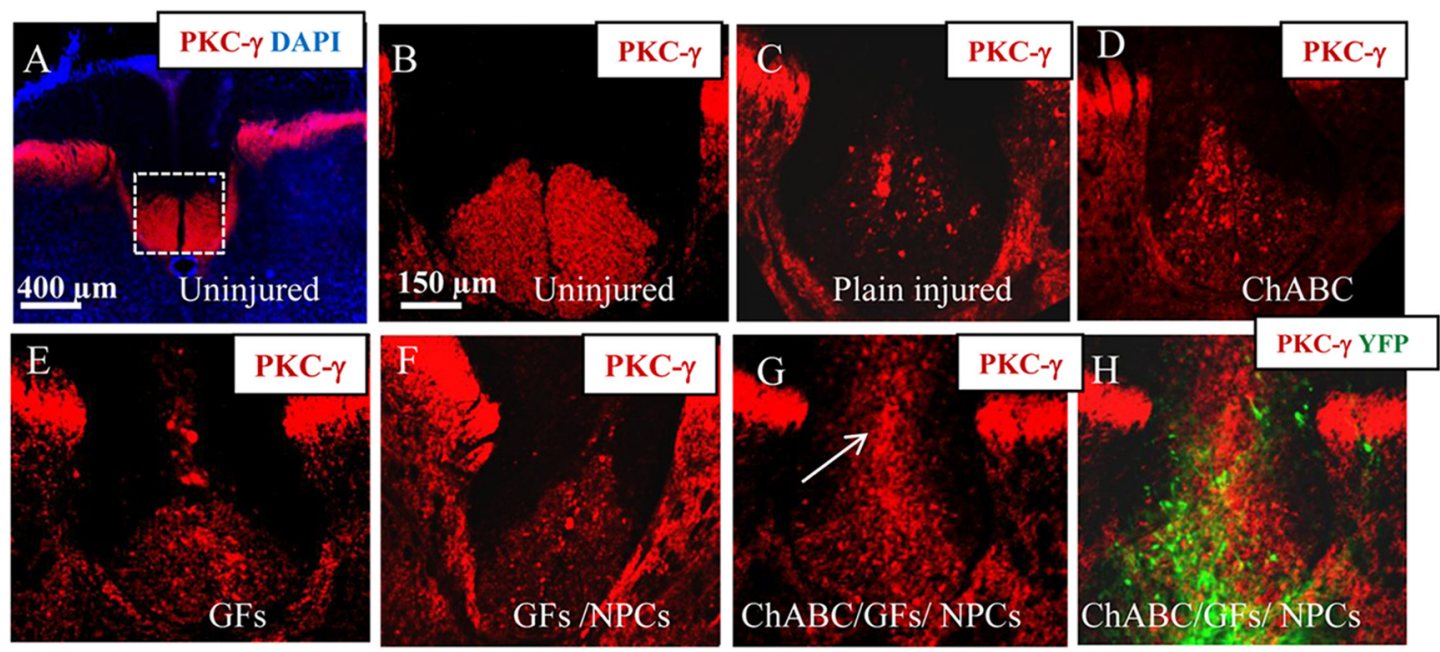

Plain Injured

ChABC

GFs

GFs / NPC

ChABC/GFs/ NPC
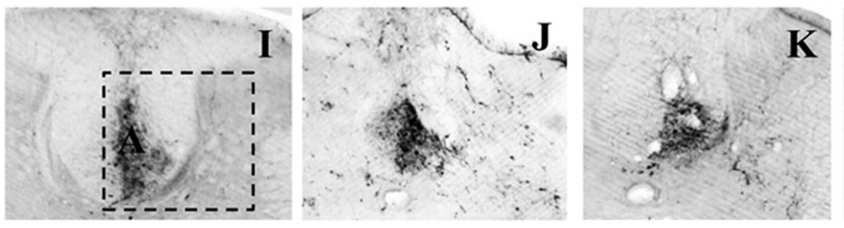

$\mathbf{K} \quad \mathbf{L}$
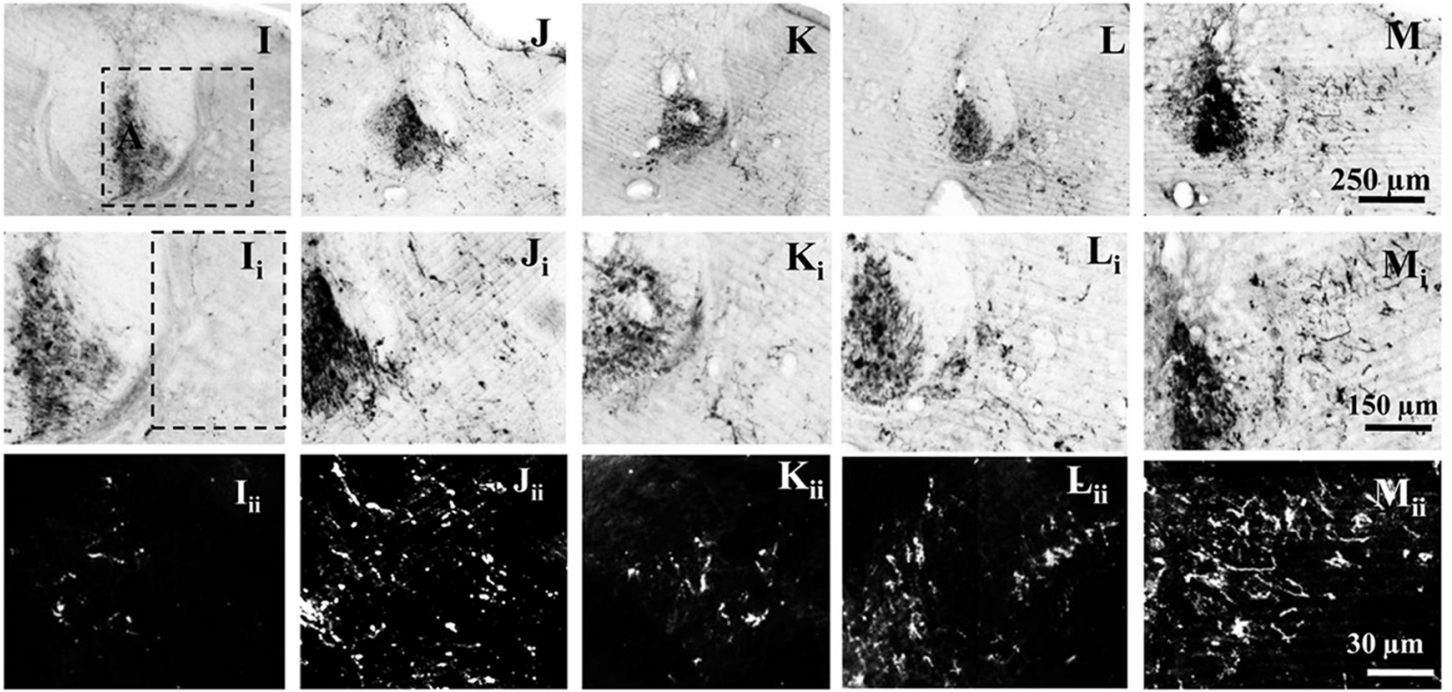

$\mathbf{N}$

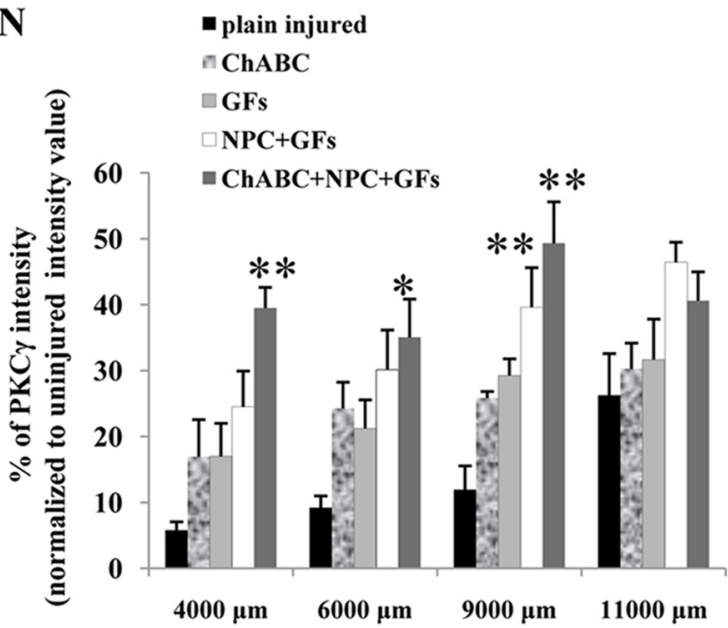

- plain injured

a ChABC

$\stackrel{9}{\stackrel{\Xi}{\Xi}}$

$\square$ GFs

$\square$ NPC+GFs

Rostral distance to injury centre

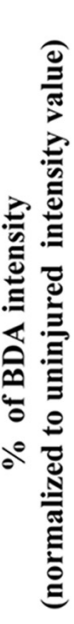

$\mathbf{O}$

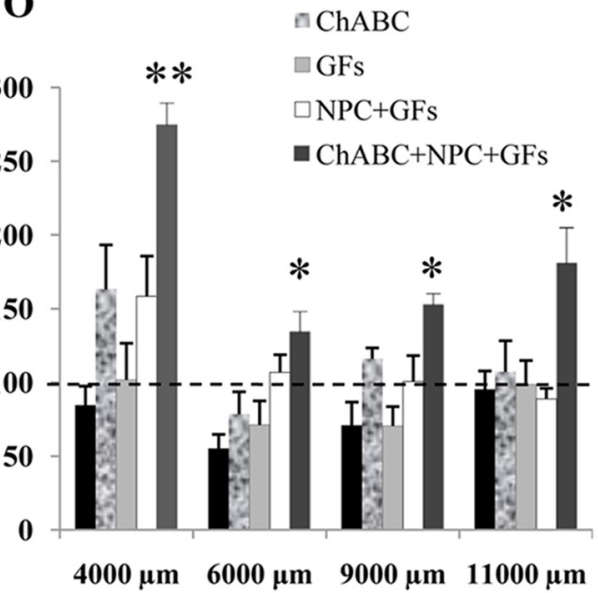

Rostral distance to injury centre

Figure 4. Combined application of ChABC, GFs, and NPCs promote axonal integrity and collateral sprouting of the CST after chronic SCl. $A, B$, Intense staining of PKC- $\gamma$ in transverse sections of uninjured spinal cord at midthoracic level shows normal localization of CST in ventral part of the dorsal columns. C, At 16 weeks after SCl, at rostral distances to injury center (data shown for 6000 $\mu \mathrm{m})$, PKC $\gamma$ immunoreactivity in the dorsal column of plain injured rats is significantly diminished, suggesting degeneration of CST. $\mathbf{C} \boldsymbol{H}$, While all injured groups showed a significant reduction in the PKC- $\gamma$ immunointensity in rostral regions to the injury center in comparison to the uninjured animals (Fig. 4), this reduction was more pronounced in the plain injured group (SCI baseline) than in our four treatment groups (ChABC, GFs, NPCs + GFs, and ChABC/GFs + NPCs). We also found evidence of PKC- $\gamma$ immunoreactivity in aberrant region of the dorsal columns of all treatment groups (arrow in $\boldsymbol{G}$ ), where the main (ST is not normally present. $\boldsymbol{N}$, Quantification of the boxed area depicted in $\boldsymbol{A}$ at various rostral distances in all experimental groups demonstrated a significant increase in PKC- $\gamma$ immunoreactivity in the combined treatment group (ChABC/GFs + NPCs) compared to the ChABC and GF groups at 4000 and $9000 \mu \mathrm{m}$ rostral to the injury (Figure legend continues.) 
CST in its target region, only the combination of these treatments promoted sufficient CST sprouting to reach significant effects.

Interestingly, no evidence of long-distance CST regeneration toward and beyond the lesion was detected (data not shown), suggesting that while the combination of ChABC/GFs + NPCs has the potential to induce local plasticity in the chronically injured spinal cord, it is not sufficient to foster long-distance axonal regeneration toward and beyond the lesion.

\section{ChABC treatment and NPC transplantation promote plasticity of serotonergic fibers in the chronically injured spinal cord}

To test whether ChABC/GF+NPC treatment exerts an effect on other pathways following SCI, we further examined the plasticity of the descending serotonergic pathway, which exerts an important neuromodulatory role in spinal cord function, including locomotion (Schmidt and Jordan, 2000; Ciranna, 2006; Jordan et al., 2008). In uninjured rats, 5-HT-positive fibers largely terminate in the ventral and intermediate horns as well as lamina $\mathrm{X}$ and to a lesser extent in the superficial layers of the dorsal horn (Fig. $5 A$ ). Interestingly, chronically injured rats in all experimental groups, including the plain injured group, demonstrated a considerable increase in serotonin immunoreactivity in rostral white matter regions compared to the uninjured group, particularly in the lateral and ventral funiculi (Fig. 5), suggesting the occurrence of spontaneous plasticity in the serotonergic pathways after compressive SCI. In the plain injured group, there was an $8.6 \pm 1.73-$ fold increase in 5-HT immunoreactivity at $1750 \mu \mathrm{m}$ to the lesion epicenter in comparison with uninjured controls (Fig. 5J). Similar results were seen at other points rostral to the lesion ranging from 3500 to $7000 \mu \mathrm{m}$ (Fig. 5J). Interestingly, comparison of serotonin immunoreactivity across the injured experimental groups revealed a robust increase in 5-HT expression in ChABC and $\mathrm{ChABC/GF}+\mathrm{NPC}$ groups (Fig. $5 J$ ). At $1750 \mu \mathrm{m}$ rostral to the lesion epicenter, ChABC and ChABC/GF+NPC groups showed $37.4 \pm 3.8$ and $31.62 \pm 8.6$-fold increase in 5-HT expression compared to the uninjured value, respectively (Fig. 5). The

\footnotetext{
(Figure legend continued.) center $\left({ }^{* *} p<0.001\right.$, one-way ANOVA, Holm-Sidak post hoc) and to the plain injured group at $6000 \mu \mathrm{m}$ rostral to injury $\left({ }^{*} p<0.01\right.$, one-way ANOVA, HolmSidak post hoc). The group that received NP(s consistently demonstrated a trend of beneficial effects that was insignificantly less than observed for the combined treatment group. While the effects seen in the NPC group were not significantly different from those seen in the $C h A B C$ and GF groups, the increases in PKC- $\gamma$ immunoreactivity reached a significant difference compared to the plain injured group at a point $9000 \mu \mathrm{m}$ rostral to the lesion ( $p<0.05$, one-way ANOVA, Holm-Sidak post hoc). Note that values depict percentages of PKC- $\gamma$ immunointensity across the injured groups that were normalized to uninjured values at the similar distances, where the uninjured value is considered $100 \%$. I-M, The BDA-labeled CST at $9000 \mu \mathrm{m}$ rostral to the lesion is demonstrated in different experimental groups after unilateral injections of BDA into the sensorimotor cortex. Images in $\boldsymbol{I}_{\boldsymbol{i}}-\boldsymbol{M}_{\boldsymbol{i}}$ show the enlarged boxes in $\boldsymbol{I}-\boldsymbol{M}$. $\boldsymbol{I}_{\boldsymbol{i j}}-\boldsymbol{M}_{i j}$, Inverted images from $\boldsymbol{I}_{\boldsymbol{i}}-\boldsymbol{M}_{\boldsymbol{i}}$ were generated from the boxed region depicted in $\boldsymbol{I}_{\boldsymbol{i}}$ for better visualization. To correct for interanimal variations in the BDA labeling efficiency, the intensity values of the BDA-labeled collaterals in the gray matter were normalized to the intensity of BDA-labeled fibers of the main CST. 0 , Quantification of the areas depicted in $\boldsymbol{I}_{\boldsymbol{i i}}-\boldsymbol{M}_{\boldsymbol{i i}}$ at various distances revealed an increase in BDA density in the combined $\mathrm{ChABC/GF}+\mathrm{NPC}$ treatment group compared to the uninjured group at all examined rostral distances to the injury center with the highest value detected at $4000 \mu \mathrm{m}$ (a 2.75-fold increase). Comparison of BDA-labeled collaterals among different injured groups also showed an increase in the density of BDA-labeled CST fibers in the ChABC/GF+NPC group that was significantly higher than all other experimental groups at 4000 and $11,000 \mu \mathrm{m}$ and to the plain injured and GF groups at 6000 and $9000 \mu \mathrm{m}$ rostral to the lesion $\left({ }^{* *} p<0.001,{ }^{*} p<0.01\right.$, one-way ANOVA, Holm-Sidak post hoc). Note that BDA density values in all injured groups are normalized to the uninjured value, where the uninjured value is considered $100 \%$
}

level of 5-HT expression in the spinal cords of the ChABC and ChABC/GF+NPC groups also remained significantly higher than all other groups at 3000 and $7000 \mu \mathrm{m}$ rostral to the lesion ${ }^{* *} p<0.001,{ }^{*} p<0.01$, one-way ANOVA, Holm-Sidak post hoc). At similar points caudal to the lesion, we also found increased serotonin immunointensity compared to the uninjured value in all experimental groups, although to a lesser extent than the effects seen rostral to the lesion. Nonetheless, the ChABCand ChABC/GF+NPC-treated groups showed 5.5- and 4.7-fold increases in serotonin immunoreactivity at $1750 \mu \mathrm{m}$ caudal to the lesion, respectively (Fig. $5 J)\left({ }^{* *} p<0.001,{ }^{*} p<0.01\right.$, one-way ANOVA, Holm-Sidak post hoc). The striking difference in the presence of serotonergic fibers between the rostral and caudal regions most likely reflects the degeneration of projecting serotonergic fibers below the level of SCI. Serotonergic fibers were also found within the NPC transplants in white matter (Fig. 5G-I). Interestingly, groups that did not receive $\mathrm{ChABC}$ showed no additional effects on axonal sprouting beyond that seen in the plain injury controls, suggesting that the increased plasticity of the serotonergic axons was principally governed by $\mathrm{ChABC}$ treatment.

\section{ChABC, GF, or NPC treatment does not enhance} neuroanatomical plasticity of pain afferents after chronic SCI Since our treatments induced sprouting of CST and serotonergic descending fibers, we also examined their effects on structural and functional plasticity of pain afferents entering the dorsal horn of spinal cord at above and below the injured spinal segments. We investigated the changes in expression of CGRP, which is involved in peripheral and spinal pain mechanisms. We quantified the sprouts of CGRP-immunoreactive fibers in laminae III-V at rostral and caudal distances ranging from 4000 to $9000 \mu \mathrm{m}$ to the lesion (Fig. 6A-F). Overall, we found increased CGRP immunoreactivity in laminae III-V of all injured groups compared to the uninjured values. Moreover, the increase in CGRP immunoreactivity was significantly higher at $9000 \mu \mathrm{m}$ both rostral and caudal to the lesion ( $22 \%$ and $84 \%$, respectively), suggesting increased sprouting of CGRP fibers in the deeper laminae of the dorsal horn following SCI. Colocalization between CGRP and GAP-43 further supported the evidence of sprouting in CGRP-positive fibers (Fig. 6G-J). Nevertheless, our analyses on different experimental treatment groups revealed no significant changes in the injury-induced pattern of sprouting in CGRP afferents in laminae III-V as the result of our treatments.

We next examined the synaptic contacts of pain afferents by examining the colocalization of CGRP and the presynaptic vesicular protein, synaptophysin (SYP). Analysis of CGRP/SYP colocalization in laminae I-V of the dorsal horn in the plain injured rats demonstrated evidence of increased synaptic terminals in CGRP-positive fibers at most points rostral or caudal to the lesion compared to uninjured values, which reached a significant level at points $6000 \mu \mathrm{m}$ and $9000 \mu \mathrm{m}$ rostral (30\% and 40\%, respectively) and caudal (49\% and $45 \%$, respectively) to the lesion (Fig. $7 A-I)$. While our results showed a trend for decreased CGRP/ SYP colocalization in the ChABC/GF+ NPC group compared to the other groups, particularly at a point $9000 \mu \mathrm{m}$ rostral to the lesion, no significant difference was noted across the experimental injured groups at any examined distance (Fig. 7I).

We further investigated plasticity in the postsynaptic transmission of pain following SCI and with the various experimental treatments by examining for changes in the expression of the immediate early gene $c$-fos in the superficial layers of the dorsal horns after a noxious heat stimulus applied to the dorsal dermatomes below the lesion. We quantified the number of $c$-fos- 
positive neurons in laminae I-II at caudal distances ranging from 4000 to $9000 \mu \mathrm{m}$ to the lesion (Fig. $8 A-F$ ). Overall, we found a significant increase in the number of $c$-fos-positive cells in laminae I-II of all injured groups compared to the uninjured values (Fig. 8). Of note, our cell quantification at $6000 \mu \mathrm{m}$ caudal to the injury site revealed a range of 3.6- to 5.25fold increase in the number of $c$-fospositive neurons in the injured groups (11-16 c-fos-positive cells) compared to the uninjured values ( $3 c$-fos-positive cells) $(p<0.05$, Student's $t$ test) (Fig. 8G). Nevertheless, consistent with our CGRP/ SYP colocalization findings, ChABC, GF, and NPC treatments alone or in combination did not lead to any significant increase in $c$-fos induction in postsynaptic nociceptive neurons of the spinal cord compared to the injury baseline control group (the plain injured rats) (Fig. $8 G$ ).

\section{ChABC treatment and NPC} transplantation improves locomotor function after chronic SCI and does not exacerbate neuropathic pain

We evaluated motor and sensory neurological outcomes after SCI and following chronic administration of the experimental treatments (Fig. 9). Hindlimb neurobehavioral recovery was assessed using the $\mathrm{BBB}$ and grid walk outcome measures. After SCI, injured rats recovered until 5 weeks after injury (before treatments) when they showed a mean BBB score of $9.6 \pm 0.39$. At week 5 , injured rats were block randomized into five experimental groups to ensure equivalent deficits across the groups (see Materials and Methods, $n=14-16$ rats/group). Our SCI baseline group (plain injured) achieved a plateau in neurobehavioral recovery at 5 weeks after injury without evidence of further recovery beyond that point. At weeks 6 and 7 (during two surgical interventions), the treatment groups showed a slight decrease in $\mathrm{BBB}$ (on average one score) compared to their pretreatment scores. While the plain injured, ChABC, GF, and NPC+GF groups did not improve after week 8 after SCI and onward, the rats treated with the combination of $\mathrm{ChABC/GFs}+\mathrm{NPC}$ regained their pretreatment scores at 2 weeks after NPC transplantation and then showed progressive improvement in their BBB scores over time. Using two-way repeated measures of ANOVA, we found a statistically significant interaction between the treatment groups and the examined time points (weeks $5-15$ after injury) ( $p<0.001$ ). Then, using the pairwise multiple-comparison method of StudentNewman-Keuls, we found statistically significant improvement in the BBB score of the ChABC/NPC+GF group $(10.96 \pm 0.63)$ at 7 weeks after transplantation (14 weeks after injury) compared to the ChABC $(9.54 \pm 0.53), \mathrm{GF}(8.96 \pm 0.46)$, and NPC $+\mathrm{GF}$ $(9 \pm 0.4)$ groups and at 8 weeks after transplantation( 15 weeks

$\mathbf{J}$
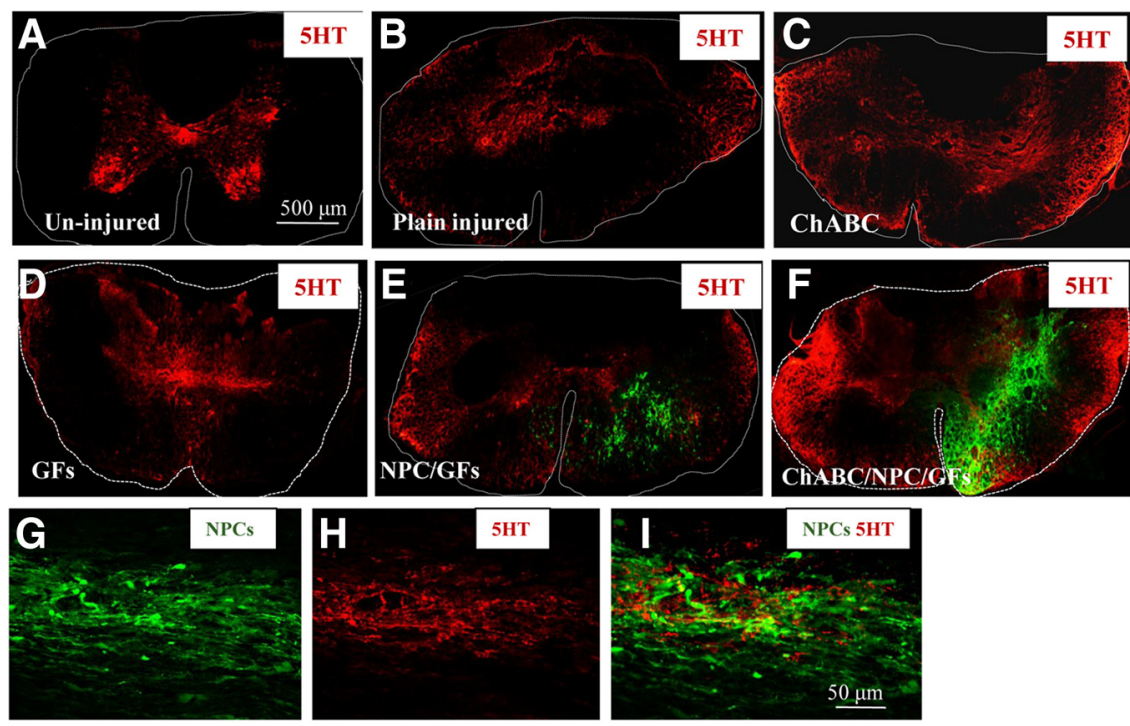

Figure 5. ChABC and NPC treatments promote plasticity of serotonergic fibers in the chronically injured spinal cord. $\boldsymbol{A}$, Transverse section of an uninjured spinal cord at midthoracic region demonstrate normal innervation pattern of the serotonergic pathway (5-HT-positive fibers) within the spinal cord. $\boldsymbol{B}-\boldsymbol{F}$, At 16 weeks after injury, 5 -HT-positive fibers in all experimental groups significant changes in their localization (images shown for $5000 \mu \mathrm{m}$ rostral). In contrast to uninjured spinal cord, 5-HTgroup at all examined distances. However, the increased sprouting of serotonergic fibers was striking in the $\mathrm{ChABC}$ and $\mathrm{ChABC}$ $\mathrm{GF}+\mathrm{NPC}$ groups compared to all other groups at 1750,3000 , and $7000 \mu \mathrm{m}$ rostral to the lesion $\left({ }^{* *} p<0.001,{ }^{*} p<0.01\right.$, one-way ivity compared to the uninjured level at points caudal to the lesion. At 1750 and $3000 \mu \mathrm{m}$, the ChABC-treated group showed a injured animals is normalized to the uninjured value, and the $y$-axis shows the fold changes in values of injured experimental groups relative to the uninjured value.

after injury, $11.15 \pm 0.60)$ relative to the plain injured $(9.77 \pm$ $0.57)$, ChABC (9.66 \pm 0.58$), \mathrm{GF}(9.25 \pm 0.49)$, and NPC/GF $(8.9 \pm 0.4)$ groups $(p<0.05$, two-way repeated-measures ANOVA followed by the pairwise multiple-comparison Student-Newman-Keuls method) (Fig. 9A). Furthermore, $\chi^{2}$ analysis revealed statistically significant greater frequency of consistent weight-supported plantar steps and evidence of forelimb-hindlimb coordination (BBB score $\geq 12$ ) in the $\mathrm{ChABC/GF}+\mathrm{NPC}$ group than in other groups from weeks 5 to 8 after transplantation $\left(\chi^{2}=52.671, \mathrm{df}=4, p<0.001\right)$ (Fig. $9 B$, data shown for week 8 ). At 8 weeks after transplantation, $39 \%$ of the animals in ChABC/NPC + GF group had a mean $\mathrm{BBB}$ score $\geq 12$, while other groups only had $7-15 \%$ of $\mathrm{BBB}$ score $\geq 12$ (Fig. 9B). 

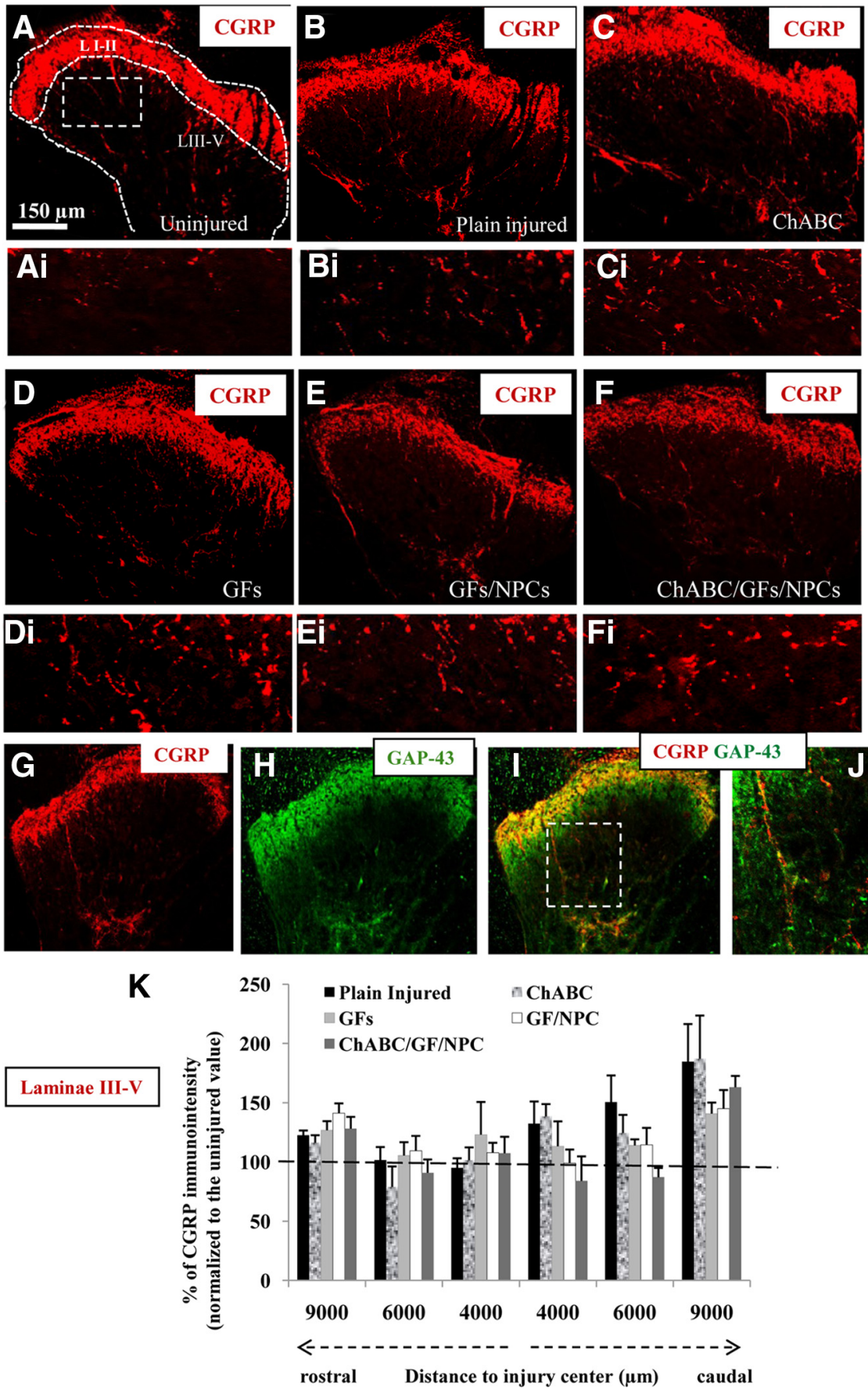

Figure 6. $C h A B C, G F$, or NPC treatment does not increase aberrant plasticity of pain afferents in the chronically injured spinal cord. Confocal immunohistochemistry for CGRP in the dorsal horn of uninjured $(\boldsymbol{A})$ and chronically injured $(\boldsymbol{B}-\boldsymbol{F})$ spinal cords in different experimental groups at $9000 \mu \mathrm{m}$ caudal to injury center is depicted. In $\boldsymbol{A}$, the traced areas identify laminae (L) I-II and III-V. In $\boldsymbol{A}_{\boldsymbol{i}}-\boldsymbol{F}_{\boldsymbol{i}}$, magnified view of an area in laminae III-V within the box in $\boldsymbol{A}$ is shown from similar areas in $\boldsymbol{A}-\boldsymbol{F}$. All injured groups demonstrated increased sprouting of (GRP-positive fibers compared to the uninjured group $\left(A_{i}\right)$. G-J, Double immunolabeling of CGRP and GAP-43 in the dorsal horn of a plain injured rat at $9000 \mu \mathrm{m}$ caudal distance to the injury site shows expression of GAP-43 in CGRP-positive afferents that further supports the evidence for sprouting of pain afferents. $\boldsymbol{K}$, At 16 weeks after injury, quantification of (GRP immunointensity in L III-V revealed an overall increase in CGRP density compared to normal value (dashed horizontal line identifying the uninjured value as 100\%) in rostral and caudal segments, which was more pronounced in $9000 \mu \mathrm{m}$. Nevertheless, no significant changes in the injury-induced pattern of sprouting in (GRP-positive afferents in laminae III-V were observed as the result of $\mathrm{ChABC}$, GF, and/or NPC treatments (one-way ANOVA). Note that (GRP density values in all injured animals are normalized to the uninjured value, where the uninjured value is considered $100 \%$.

Assessment of sensorimotor integration by grid walk analysis showed significant deficits in foot placement after SCI (Fig. 9C). While no stepping errors were observed in the baseline measurements of the rats before the SCI, at 5 weeks after injury, injured rats exhibited an average of $9.5 \pm 0.3$ footfalls per session. Consistent with the $\mathrm{BBB}$ results, at 2 weeks after treatments, most experimental groups showed an insignificant increase in their footfall errors (on average $0.98-1.2$ more errors) compared to their respective pretreatment values as the result of interventions. In contrast to all other experimental groups, the rats that received the combined treatment of $\mathrm{ChABC} / \mathrm{NPC}+\mathrm{GF}$ progressively improved following NPC transplantation. Two-way repeated-measures ANOVA revealed that the $\mathrm{ChABC} / \mathrm{NPC}+\mathrm{GF}$ group exhibited significantly fewer footfalls at 6 and 8 weeks after transplantation relative to all other groups $(p<0.05$, two-way repeated-measures ANOVA followed by the pairwise multiple-comparison Student-Newman-Keuls method) (Fig. 9C). At 8 weeks after transplantation, the $\mathrm{ChABC} / \mathrm{NPC}+\mathrm{GF}$ group showed $5.8 \pm 1$ footfall errors compared to the plain injured group with $9.6 \pm 0.73$ errors, ChABC group with $9.53 \pm 1.3$ errors, GF group with $10.5 \pm 1.3$, and GF+NPC group with $10 \pm 0.96$ errors.

We also examined mechanical and thermal allodynia using the von Frey and tail flick analyses, respectively. Mechanical sensitivity to normally non-noxious stimuli was evaluated by applying 10 stimuli using a $2 \mathrm{~g}$ von Frey filament on dorsal dermatomes above, around, and below the lesion. In our compressive model of SCI, neuropathic pain developed around 3 weeks after injury and reached a relatively plateau level with no significant changes after week 5 (Fig. 9D). No pain assessments were performed between weeks 6 and 8 due to the timing of the two consecutive surgical interventions and the required postoperative recovery period. At 2 weeks after transplantation and onward, no significant changes in mechanical sensitivity were observed in any of experimental groups compared to their pretreatment values or plain injured controls (Fig. 9D). Furthermore, no significant difference was evident across the experimental treatment groups. The onset of thermal allodynia was also observed at 3 weeks after injury, which progressively evolved over time and reached a plateau level after 9 weeks after injury (Fig. 9E). After treatments, no increased sensitivity to thermal stimulation was detected among any of the experimental groups (Fig. 9E).

\section{Discussion}

In the present study, we demonstrate for the first time the development of a combinatorial strategy that allows the successful 

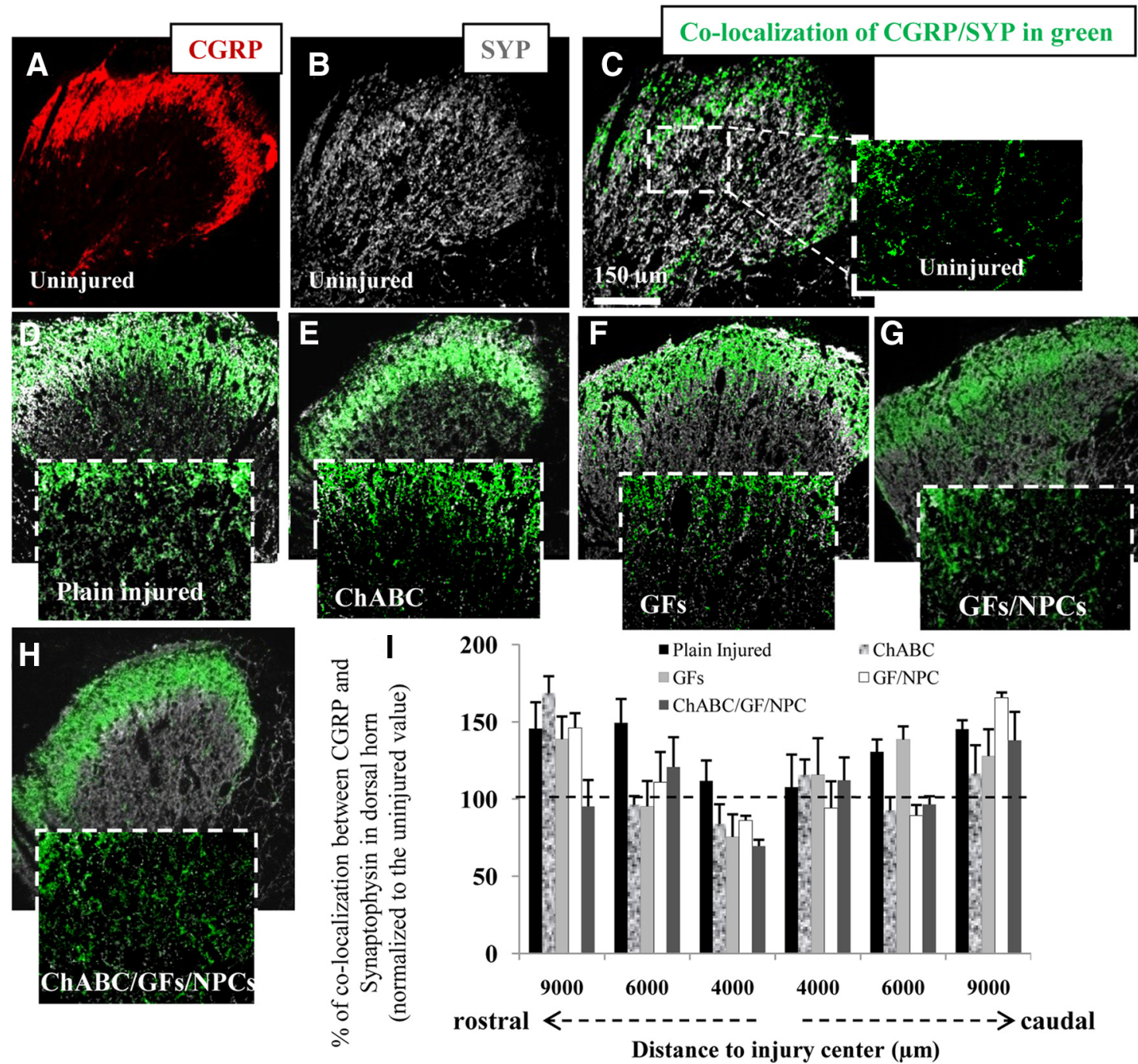

Figure 7. ChABC, GF, or NPC treatment does not exacerbate aberrant synaptic connectivity of pain afferents in the chronically injured spinal cord. The synaptic connectivity of pain afferents was determined by colocalization between CGRP and presynaptic vesicular protein, SYP. A-C, CGRP/SYP colocalization in laminae I-V of dorsal horn is depicted in dorsal horn of an uninjured rat. The image in C shows CGRP and SYP colocalized points in green on the SYP image (gray). D-H, CGRP/SYP colocalization in injured groups. I, Quantitative analysis of CGRP/SYP colocalized points in dorsal horns revealed increased synaptic terminals in CGRP-positive fibers in experimental injured groups compared to uninjured value at most examined rostral and caudal distances (images represent $9000 \mu \mathrm{m}$ (audal). While our results showed a trend for decreased (GRP/SYP colocalization in ChABC/GF +NPC group in rostral distances, no statistically significant difference was noted across the groups at any distances ( $p>0.05$, one-way ANOVA). Note that percentages of colocalization in all injured animals are normalized to the uninjured values, where the uninjured value is considered $100 \%$.

application of NPC-based therapies for the treatment of chronic SCI. This strategy, which includes oligodendroglial cell replacement using NPC transplants and optimization of the host microenvironment by disrupting the inhibitory properties of the glial scar and augmenting the deficient expression of key growth factors, successfully promoted the functional repair and plasticity of the spinal cord in a clinically relevant model of chronic compressive SCI.

While emerging evidence supports the therapeutic potential of NPC transplantation for subacute SCI (Cummings et al., 2005; Hofstetter et al., 2005; Karimi-Abdolrezaee et al., 2006; Ziv et al., 2006; Parr et al., 2008), to date no successful NPC-based application for chronic SCI has been reported. Our group (KarimiAbdolrezaee et al., 2006) and Keirstead et al. (2005) have previously reported on the challenges associated with cell transplantation in the microenvironment of chronic SCI. We demonstrated that the therapeutic efficiency of NPC transplants dramatically declines in the chronically injured spinal cord
(Karimi-Abdolrezaee et al., 2006) as the majority of NPCs fail to disperse and integrate within the host tissue, leading to the suboptimal survival of NPCs. Keirstead et al. (2005) also demonstrated that transplanted oligodendrocyte precursor cells failed to promote remyelination or functional locomotor recovery in a chronic model of SCI. These observations suggest that the microenvironment of the chronically injured spinal cord restricts the reparative capacities of engrafted stem cells.

Here, we demonstrate that CSPGs in the postinjury glial scar play a key role in the suboptimal outcomes of NPC transplantation in chronic SCI. In the areas of transplantation, deposits of CSPGs associated with astrocytes surround the clustered nonviable NPCs. Interestingly, degradation of CSPGs by the transient delivery of ChABC before the NPC transplantation resulted in a marked increase in the long-term survival of NPCs in the chronically injured spinal cord. While the exact molecular mechanisms by which CSPGs restrict NPCs require further elucidation, our results indicate that CSPGs may interfere with the integration 

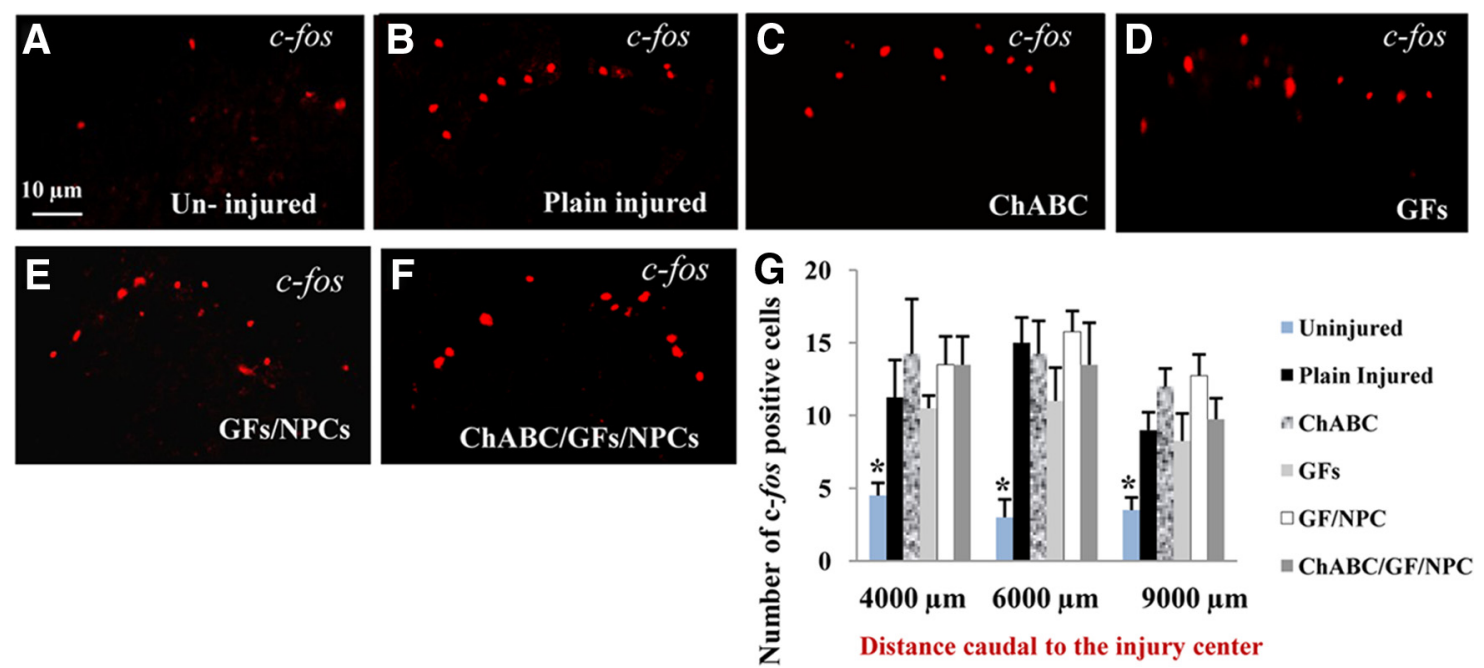

Figure 8. ChABC, GF, or NPC treatment does not enhance postsynaptic transmission of pain in chronically injured spinal cord. $\boldsymbol{A}-\boldsymbol{F}$, Transverse sections of spinal cord show the expression of the immediate early gene c-fos in superficial layers of dorsal horns after a noxious heat stimuli applied to the dermatomes below the lesion. $\mathbf{G}$, The number of $c$-fos-positive neurons in the superficial layers of dorsal horns in all injured rats regardless of their treatments was increased compared to uninjured animals at all examined caudal distances ( $p<0.05$, one-way ANOVA). However, no significant difference was observed among the injured/treated groups ( $p>0.05$, one-way ANOVA).

and migration of NPCs. CSPGs may also prevent the interactions of NPCs with the host axons in the injured white matter tracts where surviving NPCs mainly integrate and differentiate into oligodendrocytes. Interestingly, consistent with our previous reports (Karimi-Abdolrezaee et al., 2006; Eftekharpour et al., 2007), the presence of GFs in this therapeutic combination efficiently directed the fate of undifferentiated NPCs toward an oligodendroglial lineage, and $\mathrm{ChABC}$ treatment did not alter the differentiation pattern of engrafted NPCs. One previous study (Ikegami et al., 2005) also showed the positive effects of ChABC treatment on migration of NPCs in a subacute stage of SCI. However, no structural or functional assessments were performed to determine the effects of ChABC on NPC cell survival and differentiation or their therapeutic potential for promoting neuroanatomical and functional recovery after subacute transplantation.

Here, we have provided mechanistic insights into the effects of ChABC, GFs, and NPCs alone or in combination in the chronically injured spinal cord. Of note, this strategy significantly increased the axonal density in the main CST as well as collateral sprouting of CST fibers within the target gray matter in rostral regions to the injury center. The observed increase in axonal density in the main CST may be attributed to enhanced axonal preservation and/or axonal sprouting. Interestingly, while each treatment individually showed a trend toward promoting axonal integrity/sprouting of the CST in the chronically injured spinal cord, only their combination was able to achieve a significant effect, emphasizing on the importance of this combinatorial approach. Several known beneficial effects mediated by ChABC, GFs, and/or NPCs may have collectively contributed to this neuroanatomical improvement. Enhanced axonal ensheathment and/or remyelination of the CST axons by GFs or NPC-derived oligodendrocytes may have played a role (Hofstetter et al., 2005; Keirstead et al., 2005; Karimi-Abdolrezaee et al., 2006). Additionally, modification of the extracellular matrix by ChABC treatment may have induced axonal sprouting of the CST as previously shown in descending and ascending spinal cord tracts (Barritt et al., 2006; Houle et al., 2006; Massey et al., 2006). Furthermore, recent studies have shown that macrophages actively induce axonal retraction/die-back in the sensory axons (Horn et al., 2008), and interestingly, ChABC treatment efficiently atten- uates the macrophage-mediated axonal retraction (Busch et al., 2009). Nonetheless, further investigation with intermediate time points is required to temporally and spatially elucidate the effects of each treatment on the integrity and plasticity of the CST.

The combinatorial strategy of ChABC, GFs, and NPCs also significantly increased the collateral sprouting of the CST fibers within the target gray matter in the rostral segments to the lesion. It has been shown that the increased collateral sprouting of the CST effectively contributes to the establishment of new connections leading to enhanced recovery of neurological function after SCI (Weidner et al., 2001; Bareyre et al., 2004). Interestingly, despite marked increase in local sprouting of the CST at points rostral to the lesion; we found no evidence of long-distance regeneration of the CST fibers toward and below the lesion site. This may be related to different factors, including the significant length of axonal die-back in the CST in the chronically injured spinal cord (Seif et al., 2007), the extended central cavity within the chronic lesion that dramatically disrupts the integrity of the CST (Thuret et al., 2006), the presence of myelin debris (Schwab and Bartholdi, 1996; Schwab, 2002, 2004) and/or macrophages (Horn et al., 2008; Busch et al., 2009) that pose a barrier to regenerating axons around the lesion, and the residual CSPGs that have been unaffected by our ChABC treatment.

This combinatorial treatment also remarkably promoted axonal sprouting in the spinal serotonergic pathway, which plays a major role in modulating various neuronal activities, including locomotion, in the spinal cord (Schmidt and Jordan, 2000; Ciranna, 2006; Jordan et al., 2008). Previous studies demonstrated that increased sprouting of serotonergic fibers in the injured spinal cord leads to improved recovery of locomotion ( $\mathrm{Li}$ and Strittmatter, 2003; Li et al., 2004, 2005). Interestingly, our data suggest that $\mathrm{ChABC}$ treatment played the major role in enhancing plasticity of the serotonergic fibers. We found that ChABC treatment alone had similar significant effects as the combined treatment with ChABC/GFs + NPCs, whereas GFs and NPCs alone did not show any additive effects. This is in agreement with previous work that implicated acute treatment of ChABC in inducing neuroanatomical plasticity of the serotonergic fibers (Barritt et al., 2006). Collectively, our data demonstrate that while treatment with ChABC, GFs, or NPCs alone exerts some beneficial neuroana- 
tomical effects by attenuating the inhibitory effects of CSPGs in postinjury glial scar, enhancing oligodendroglial replacement and remyelination as well as promoting the integrity and/or plasticity of the CST and serotonergic pathways, only their combined application resulted in significant recovery of locomotor function after chronic SCI.

We also demonstrate that the combination of ChABC, GFs, and NPCs does not enhance the injury-induced plasticity of pain afferents in the dorsal horn of the spinal cord, and importantly does not lead to increased neuropathic pain. Consistent with previous studies (Bennett et al., 2000; Weaver et al., 2001; Bruce et al., 2002; Ondarza et al., 2003), we show that increased sprouting and synaptic connectivity of CGRP-positive pain afferents appears to partly mediate the development of chronic neuropathic pain after SCI. Nevertheless, treatment with ChABC, GF, or NPC does not exacerbate this process. This is particularly important in the light of recent report that suggests a correlation between induction of allodynia after SCI and the transplantation of NPCs that predominantly differentiated into astrocytes (Hofstetter et al., 2005). Hofstetter et al. (2005) reported that transplants of naive NPCs principally differentiate into astrocytes, which was associated with induced aberrant sprouting of CGRP fibers rostral to the injury, leading to increased allodynia. These authors provide further evidence that these adverse effects can be prevented if oligodendroglial differentiation is enhanced. In our transplantation protocol, $>70 \%$ of NPCs differentiate into oligodendrocytes; therefore, we did not anticipate observing increased pain sensitivity as the result of NPC transplantation. ChABC or GF therapy also did not exacerbate the injury-induced sprouting of CGRP pain fibers or the synaptic connectivity of these fibers, supporting the safety of our combinatorial approach.

In conclusion, the present study emphasized a key role for CSPGs in restricting the reparative capacities of NPCs in the chronically injured spinal cord, as ChABC pretreatment dramatically optimizes the outcomes of NPC transplantation. Here, we have identified multiple mechanisms by which the combination of ChABC, GFs, and NPCs may have improved functional recovery after chronic SCI: (1) robust NPC-derived oligodendroglial replacement associated with axonal ensheathment and remyelination; (2) diminished axonal die-back and/or increased axonal sprouting in the CST; and (3) increased sprouting of serotonergic fibers. Importantly, this combinatorial ap-

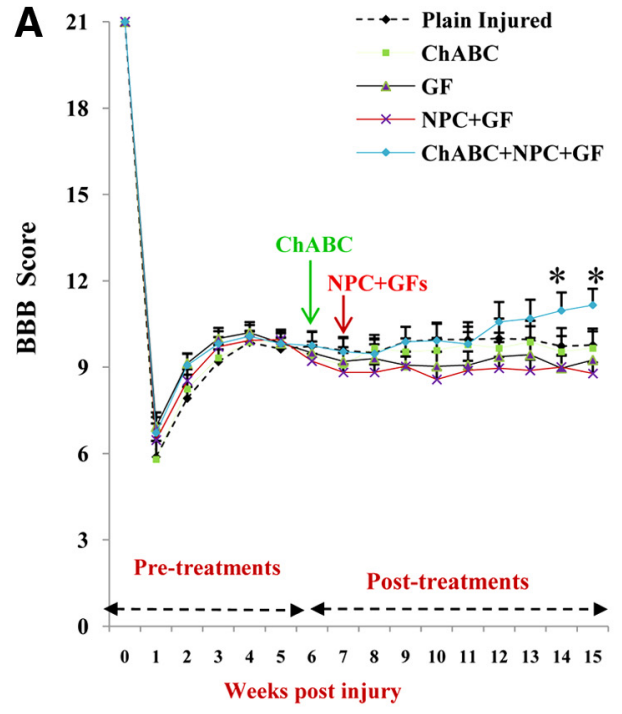

C

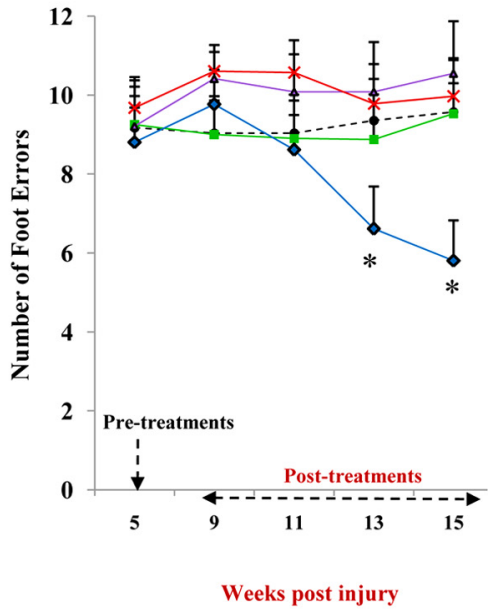

B
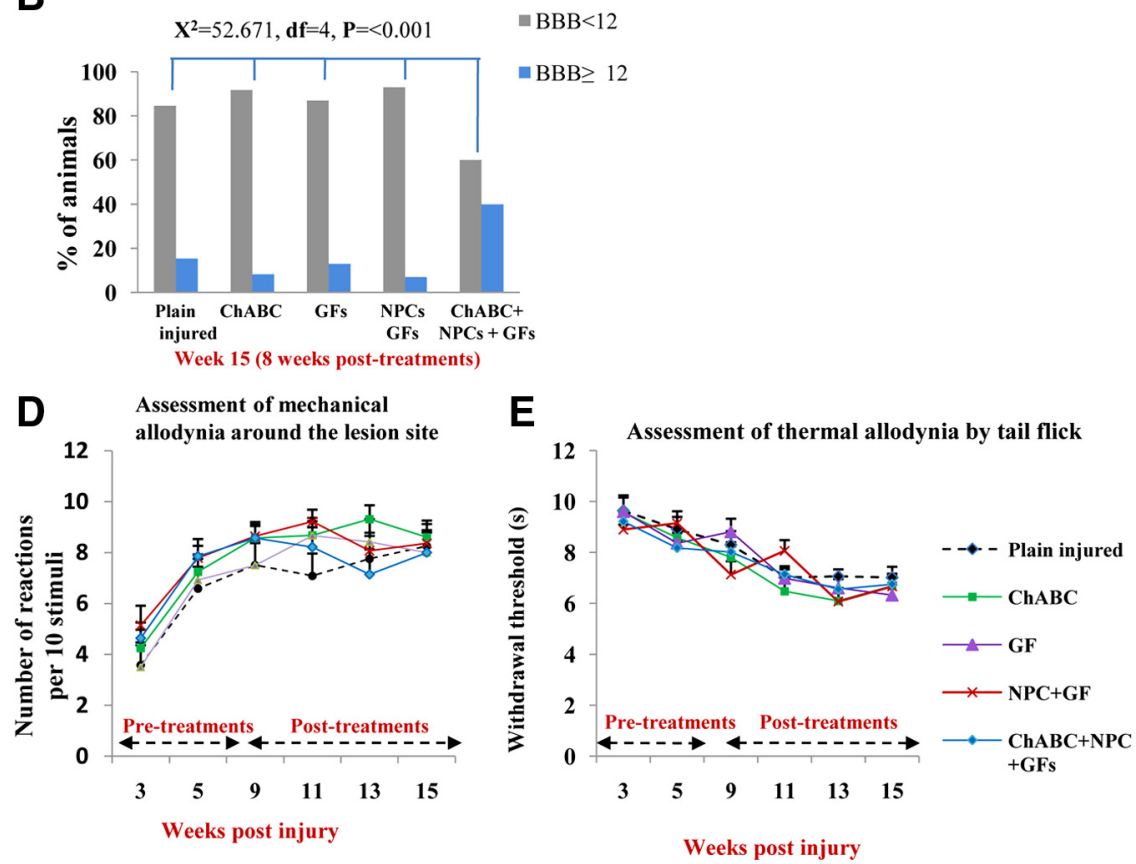

Figure 9. Combination of ChABC treatment with GFs and NPC transplant improves locomotor function without exacerbating neuropathic pain after chronic SCI. $A$, Hindlimb locomotion was assessed by the BBB open field scale in plain injured rats $(n=13)$ and in rats with SCI treated with ChABC $(n=13)$, the GFs EGF, bFGF, and PDGF-AA $(n=15)$, GFs +NPCs $(n=14)$, and ChABC/GFs + NPCs $(n=14)$ over a 15 week postinjury period ( 8 weeks after NPC transplantation). At 5 weeks after injury, before starting the treatments, all chronically injured rats were block randomized into the above treatment groups. During weeks 6 and 7 , rats in all groups, except the plain injured group, underwent two consecutive treatment surgeries. While the rats receiving ChABC, GFs, or NPCs + GFs showed no further improvement in the BBB scores after achieving a plateau at 8 weeks, the rats receiving the combination of ChABC/GFs + NPCs showed a progressive improvement in neurobehavioral recovery commencing at week 12 and continuing onward to weeks 14 and 15, at which point the extent of improvement reached a significant level compared to the ChABC, GFs, and NPCs + GFs (week 14) and to all other groups (week 15) ( $p<0.05$, two-way repeated-measures ANOVA followed by the pairwise multiple-comparison post hoc Student-Newman-Keuls method). $\boldsymbol{B}, \chi^{2}$ analysis revealed statistically significant greater frequency of consistent weight-supported plantar steps and occasional forelimb-hindlimb coordination (BBB score $\geq 12$ ) in the ChABC/GF+NPC group compared to the other groups from 5 to 8 weeks after transplantation (data shown for 8 weeks after transplantation). C, Grid walk analysis undertaken in the same groups of animals described in $\boldsymbol{A}$ showed fewer errors in hindlimb placements at 6 and 8 weeks after transplantation (13 and 15 weeks after injury) in the ChABC/GF + NPC group when compared to the other groups ( $p<0.05$, two-way repeated-measures ANOVA followed by the pairwise multiple-comparison post hoc Student-Newman-Keuls method). $\boldsymbol{D}$, Von Frey analysis of the dermatomes above and below the lesion in the same animals described in $\boldsymbol{A}$ demonstrated that mechanical allodynia developed in all groups following $\mathrm{SCl}$ and plateaued at 9 weeks. None of the treatments exacerbated the extent of mechanical allodynia in comparison with the plain injured controls. $\boldsymbol{E}$, Thermal allodynia also developed following $\mathrm{SCl}$ and reached a plateau level after 9 weeks after injury. None of the treatments exacerbated thermal allodynia in comparison with the plain injured controls. 
proach did not promote aberrant plasticity of pain afferents or neuropathic pain. Our report is the first to demonstrate a successful and safe NPC-mediated repair strategy for the treatment of chronic SCI. These findings may facilitate the clinical application of NPCs for patients suffering from debilitating chronic SCI.

\section{References}

Alvarez-Buylla A, Temple S (1998) Stem cells in the developing and adult nervous system. J Neurobiol 36:105-110.

Asher RA, Morgenstern DA, Fidler PS, Adcock KH, Oohira A, Braistead JE, Levine JM, Margolis RU, Rogers JH, Fawcett JW (2000) Neurocan is upregulated in injured brain and in cytokine-treated astrocytes. J Neurosci 20:2427-2438.

Bareyre FM, Kerschensteiner M, Raineteau O, Mettenleiter TC, Weinmann O, Schwab ME (2004) The injured spinal cord spontaneously forms a new intraspinal circuit in adult rats. Nat Neurosci 7:269-277.

Barritt AW, Davies M, Marchand F, Hartley R, Grist J, Yip P, McMahon SB, Bradbury EJ (2006) Chondroitinase $\mathrm{ABC}$ promotes sprouting of intact and injured spinal systems after spinal cord injury. J Neurosci 26:10856-10867.

Basso DM, Beattie MS, Bresnahan JC (1995) A sensitive and reliable locomotor rating scale for open field testing in rats. J Neurotrauma 12:1-21.

Bennett AD, Chastain KM, Hulsebosch CE (2000) Alleviation of mechanical and thermal allodynia by CGRP $(8-37)$ in a rodent model of chronic central pain. Pain 86:163-175.

Biernaskie J, Sparling JS, Liu J, Shannon CP, Plemel JR, Xie Y, Miller FD, Tetzlaff W (2007) Skin-derived precursors generate myelinating Schwann cells that promote remyelination and functional recovery after contusion spinal cord injury. J Neurosci 27:9545-9559.

Bradbury EJ, Moon LD, Popat RJ, King VR, Bennett GS, Patel PN, Fawcett JW, McMahon SB (2002) Chondroitinase ABC promotes functional recovery after spinal cord injury. Nature 416:636-640.

Bruce JC, Oatway MA, Weaver LC (2002) Chronic pain after clipcompression injury of the rat spinal cord. Exp Neurol 178:33-48.

Busch SA, Horn KP, Silver DJ, Silver J (2009) Overcoming macrophagemediated axonal dieback following CNS injury. J Neurosci 29:9967-9976.

Cafferty WB, Yang SH, Duffy PJ, Li S, Strittmatter SM (2007) Functional axonal regeneration through astrocytic scar genetically modified to digest chondroitin sulfate proteoglycans. J Neurosci 27:2176-2185.

Ciranna L (2006) Serotonin as a modulator of glutamate- and GABAmediated neurotransmission: implications in physiological functions and in pathology. Curr Neuropharmacol 4:101-114.

Cummings BJ, Uchida N, Tamaki SJ, Salazar DL, Hooshmand M, Summers R, Gage FH, Anderson AJ (2005) Human neural stem cells differentiate and promote locomotor recovery in spinal cord-injured mice. Proc Natl Acad Sci U S A 102:14069-14074.

Eftekharpour E, Karimi-Abdolrezaee S, Wang J, El Beheiry H, Morshead C, Fehlings MG (2007) Myelination of congenitally dysmyelinated spinal cord axons by adult neural precursor cells results in formation of nodes of Ranvier and improved axonal conduction. J Neurosci 27:3416-3428.

Fehlings MG, Tator CH (1995) The relationships among the severity of spinal cord injury, residual neurological function, axon counts, and counts of retrogradely labeled neurons after experimental spinal cord injury. Exp Neurol 132:220-228.

Fleming JC, Bao F, Chen Y, Hamilton EF, Relton JK, Weaver LC (2008) Alpha4beta1 integrin blockade after spinal cord injury decreases damage and improves neurological function. Exp Neurol 214:147-159.

Fouad K, Schnell L, Bunge MB, Schwab ME, Liebscher T, Pearse DD (2005) Combining Schwann cell bridges and olfactory-ensheathing glia grafts with chondroitinase promotes locomotor recovery after complete transection of the spinal cord. J Neurosci 25:1169-1178.

Goldman S (2005) Stem and progenitor cell-based therapy of the human central nervous system. Nat Biotechnol 23:862-871.

Gris D, Marsh DR, Oatway MA, Chen Y, Hamilton EF, Dekaban GA, Weaver LC (2004) Transient blockade of the CD11d/CD18 integrin reduces secondary damage after spinal cord injury, improving sensory, autonomic, and motor function. J Neurosci 24:4043-4051.

Hofstetter CP, Holmström NA, Lilja JA, Schweinhardt P, Hao J, Spenger C, Wiesenfeld-Hallin Z, Kurpad SN, Frisén J, Olson L (2005) Allodynia limits the usefulness of intraspinal neural stem cell grafts; directed differentiation improves outcome. Nat Neurosci 8:346-353.

Horn KP, Busch SA, Hawthorne AL, van Rooijen N, Silver J (2008) Another barrier to regeneration in the CNS: activated macrophages induce extensive retraction of dystrophic axons through direct physical interactions. J Neurosci 28:9330-9341.

Horner PJ, Power AE, Kempermann G, Kuhn HG, Palmer TD, Winkler J, Thal LJ, Gage FH (2000) Proliferation and differentiation of progenitor cells throughout the intact adult rat spinal cord. J Neurosci 20:2218 -2228.

Houle JD, Tom VJ, Mayes D, Wagoner G, Phillips N, Silver J (2006) Combining an autologous peripheral nervous system "bridge" and matrix modification by chondroitinase allows robust, functional regeneration beyond a hemisection lesion of the adult rat spinal cord. J Neurosci 26:7405-7415.

Ikegami T, Nakamura M, Yamane J, Katoh H, Okada S, Iwanami A, Watanabe K, Ishii K, Kato F, Fujita H, Takahashi T, Okano HJ, Toyama Y, Okano H (2005) Chondroitinase ABC combined with neural stem/progenitor cell transplantation enhances graft cell migration and outgrowth of growthassociated protein-43-positive fibers after rat spinal cord injury. Eur J Neurosci 22:3036-3046.

Jones LL, Margolis RU, Tuszynski MH (2003) The chondroitin sulfate proteoglycans neurocan, brevican, phosphacan, and versican are differentially regulated following spinal cord injury. Exp Neurol 182:399-411.

Jordan LM, Liu J, Hedlund PB, Akay T, Pearson KG (2008) Descending command systems for the initiation of locomotion in mammals. Brain Res Rev 57:183-191.

Karimi-Abdolrezaee S, Eftekharpour E, Fehlings MG (2004) Temporal and spatial patterns of Kv1.1 and Kv1.2 protein and gene expression in spinal cord white matter after acute and chronic spinal cord injury in rats: implications for axonal pathophysiology after neurotrauma. Eur J Neurosci 19:577-589.

Karimi-Abdolrezaee S, Eftekharpour E, Wang J, Morshead CM, Fehlings MG (2006) Delayed transplantation of adult neural stem cells promotes remyelination and functional recovery after spinal cord injury. J Neurosci 26:3377-3389.

Keirstead HS, Nistor G, Bernal G, Totoiu M, Cloutier F, Sharp K, Steward O (2005) Human embryonic stem cell-derived oligodendrocyte progenitor cell transplants remyelinate and restore locomotion after spinal cord injury. J Neurosci 25:4694-4705.

Li S, Strittmatter SM (2003) Delayed systemic Nogo-66 receptor antagonist promotes recovery from spinal cord injury. J Neurosci 23:4219-4227.

Li S, Liu BP, Budel S, Li M, Ji B, Walus L, Li W, Jirik A, Rabacchi S, Choi E, Worley D, Sah DW, Pepinsky B, Lee D, Relton J, Strittmatter SM (2004) Blockade of Nogo-66, myelin-associated glycoprotein, and oligodendrocyte myelin glycoprotein by soluble Nogo-66 receptor promotes axonal sprouting and recovery after spinal injury. J Neurosci 24:10511-10520.

Li S, Kim JE, Budel S, Hampton TG, Strittmatter SM (2005) Transgenic inhibition of Nogo-66 receptor function allows axonal sprouting and improved locomotion after spinal injury. Mol Cell Neurosci 29:26-39.

Lu P, Jones LL, Snyder EY, Tuszynski MH (2003) Neural stem cells constitutively secrete neurotrophic factors and promote extensive host axonal growth after spinal cord injury. Exp Neurol 181:115-129.

Massey JM, Hubscher CH, Wagoner MR, Decker JA, Amps J, Silver J, Onifer SM (2006) Chondroitinase ABC digestion of the perineuronal net promotes functional collateral sprouting in the cuneate nucleus after cervical spinal cord injury. J Neurosci 26:4406-4414.

Massey JM, Amps J, Viapiano MS, Matthews RT, Wagoner MR, Whitaker CM, Alilain W, Yonkof AL, Khalyfa A, Cooper NG, Silver J, Onifer SM (2008) Increased chondroitin sulfate proteoglycan expression in denervated brainstem targets following spinal cord injury creates a barrier to axonal regeneration overcome by chondroitinase $\mathrm{ABC}$ and neurotrophin-3. Exp Neurol 209:426-445.

Morgenstern DA, Asher RA, Fawcett JW (2002) Chondroitin sulphate proteoglycans in the CNS injury response. Prog Brain Res 137:313-332.

Mori M, Kose A, Tsujino T, Tanaka C (1990) Immunocytochemical localization of protein kinase $\mathrm{C}$ subspecies in the rat spinal cord: light and electron microscopic study. J Comp Neurol 299:167-177.

Morshead CM, Reynolds BA, Craig CG, McBurney MW, Staines WA, Morassutti D, Weiss S, van der Kooy D (1994) Neural stem cells in the adult mammalian forebrain: a relatively quiescent subpopulation of subependymal cells. Neuron 13:1071-1082.

Nashmi R, Fehlings MG (2001) Changes in axonal physiology and morphology after chronic compressive injury of the rat thoracic spinal cord. Neuroscience 104:235-251.

Oatway MA, Chen Y, Weaver LC (2004) The 5-HT3 receptor facilitates at- 
level mechanical allodynia following spinal cord injury. Pain 110: 259-268.

Oatway MA, Chen Y, Bruce JC, Dekaban GA, Weaver LC (2005) AntiCD11d integrin antibody treatment restores normal serotonergic projections to the dorsal, intermediate, and ventral horns of the injured spinal cord. J Neurosci 25:637-647.

Ondarza AB, Ye Z, Hulsebosch CE (2003) Direct evidence of primary afferent sprouting in distant segments following spinal cord injury in the rat: colocalization of GAP-43 and CGRP. Exp Neurol 184:373-380.

Parr AM, Kulbatski I, Zahir T, Wang X, Yue C, Keating A, Tator CH (2008) Transplanted adult spinal cord-derived neural stem/progenitor cells promote early functional recovery after rat spinal cord injury. Neuroscience 155:760-770.

Reynolds BA, Weiss S (1992) Generation of neurons and astrocytes from isolated cells of the adult mammalian central nervous system. Science 255:1707-1710.

Rivlin AS, Tator CH (1978) Effect of duration of acute spinal cord compression in a new acute cord injury model in the rat. Surg Neurol 10:38-43.

Schmidt BJ, Jordan LM (2000) The role of serotonin in reflex modulation and locomotor rhythm production in the mammalian spinal cord. Brain Res Bull 53:689-710.

Schwab ME (2002) Repairing the injured spinal cord. Science 295:1029_ 1031.

Schwab ME (2004) Nogo and axon regeneration. Curr Opin Neurobiol 14:118-124.

Schwab ME, Bartholdi D (1996) Degeneration and regeneration of axons in the lesioned spinal cord. Physiol Rev 76:319-370.

Seif GI, Nomura H, Tator CH (2007) Retrograde axonal degeneration "dieback" in the corticospinal tract after transection injury of the rat spinal cord: a confocal microscopy study. J Neurotrauma 24:1513-1528.

Silver J, Miller JH (2004) Regeneration beyond the glial scar. Nat Rev Neurosci 5:146-156.
Tang X, Davies JE, Davies SJ (2003) Changes in distribution, cell associations, and protein expression levels of NG2, neurocan, phosphacan, brevican, versican V2, and tenascin-C during acute to chronic maturation of spinal cord scar tissue. J Neurosci Res 71:427-444.

Tang XQ, Tanelian DL, Smith GM (2004) Semaphorin3A inhibits nerve growth factor-induced sprouting of nociceptive afferents in adult rat spinal cord. J Neurosci 24:819-827.

Tang XQ, Heron P, Mashburn C, Smith GM (2007) Targeting sensory axon regeneration in adult spinal cord. J Neurosci 27:6068-6078.

Teng YD, Lavik EB, Qu X, Park KI, Ourednik J, Zurakowski D, Langer R, Snyder EY (2002) Functional recovery following traumatic spinal cord injury mediated by a unique polymer scaffold seeded with neural stem cells. Proc Natl Acad Sci U S A 99:3024-3029.

Teng YD, Liao WL, Choi H, Konya D, Sabharwal S, Langer R, Sidman RL, Snyder EY, Frontera WR (2006) Physical activity-mediated functional recovery after spinal cord injury: potential roles of neural stem cells. Regen Med 1:763-776.

Thuret S, Moon LD, Gage FH (2006) Therapeutic interventions after spinal cord injury. Nat Rev Neurosci 7:628-643.

Tropepe V, Sibilia M, Ciruna BG, Rossant J, Wagner EF, van der Kooy D (1999) Distinct neural stem cells proliferate in response to EGF and FGF in the developing mouse telencephalon. Dev Biol 208:166-188.

Weaver LC, Verghese P, Bruce JC, Fehlings MG, Krenz NR, Marsh DR (2001) Autonomic dysreflexia and primary afferent sprouting after clipcompression injury of the rat spinal cord. J Neurotrauma 18:1107-1119.

Weidner N, Ner A, Salimi N, Tuszynski MH (2001) Spontaneous corticospinal axonal plasticity and functional recovery after adult central nervous system injury. Proc Natl Acad Sci U S A 98:3513-3518.

Ziv Y, Avidan H, Pluchino S, Martino G, Schwartz M (2006) Synergy between immune cells and adult neural stem/progenitor cells promotes functional recovery from spinal cord injury. Proc Natl Acad Sci U S A 103:13174-13179. 